\title{
Evolutionary Programming Made Faster
}

\author{
Xin Yao, Senior Member, IEEE, Yong Liu, Student Member, IEEE, and Guangming Lin
}

\begin{abstract}
Evolutionary programming (EP) has been applied with success to many numerical and combinatorial optimization problems in recent years. EP has rather slow convergence rates, however, on some function optimization problems. In this paper, a "fast EP" (FEP) is proposed which uses a Cauchy instead of Gaussian mutation as the primary search operator. The relationship between FEP and classical EP (CEP) is similar to that between fast simulated annealing and the classical version. Both analytical and empirical studies have been carried out to evaluate the performance of FEP and CEP for different function optimization problems. This paper shows that FEP is very good at search in a large neighborhood while CEP is better at search in a small local neighborhood. For a suite of 23 benchmark problems, FEP performs much better than CEP for multimodal functions with many local minima while being comparable to CEP in performance for unimodal and multimodal functions with only a few local minima. This paper also shows the relationship between the search step size and the probability of finding a global optimum and thus explains why FEP performs better than CEP on some functions but not on others. In addition, the importance of the neighborhood size and its relationship to the probability of finding a near-optimum is investigated. Based on these analyses, an improved FEP (IFEP) is proposed and tested empirically. This technique mixes different search operators (mutations). The experimental results show that IFEP performs better than or as well as the better of FEP and CEP for most benchmark problems tested.
\end{abstract}

Index Terms - Cauchy mutations, evolutionary programming, mixing operators.

\section{INTRODUCTION}

A LTHOUGH evolutionary programming (EP) was first proposed as an approach to artificial intelligence [1], it has been recently applied with success to many numerical and combinatorial optimization problems [2]-[4]. Optimization by EP can be summarized into two major steps:

1) mutate the solutions in the current population;

2) select the next generation from the mutated and the current solutions.

These two steps can be regarded as a population-based version of the classical generate-and-test method [5], where mutation is

Manuscript received October 30, 1996; revised February 3, 1998, August 14, 1998, and January 7, 1999. This work was supported in part by the Australian Research Council through its small grant scheme and by a special research grant from the University College, UNSW, ADFA.

$\mathrm{X}$. Yao was with the Computational Intelligence Group, School of Computer Science, University College, The University of New South Wales, Australian Defence Force Academy, Canberra, ACT, Australia 2600. He is now with the School of Computer Science, University of Birmingham, Birmingham B15 2TT U.K. (e-mail: X.Yao@cs.bham.ac.uk).

Y. Liu and G. Lin are with the Computational Intelligence Group, School of Computer Science, University College, The University of New South Wales, Australian Defence Force Academy, Canberra, ACT, Australia 2600 (e-mail: liuy@cs.adfa.edu.au; glin@cs.adfa.edu.au).

Publisher Item Identifier S 1089-778X(99)04556-7. used to generate new solutions (offspring) and selection is used to test which of the newly generated solutions should survive to the next generation. Formulating EP as a special case of the generate-and-test method establishes a bridge between EP and other search algorithms, such as evolution strategies, genetic algorithms, simulated annealing (SA), tabu search (TS), and others, and thus facilitates cross-fertilization among different research areas.

One disadvantage of EP in solving some of the multimodal optimization problems is its slow convergence to a good near-optimum (e.g., $f_{8}$ to $f_{13}$ studied in this paper). The generate-and-test formulation of EP indicates that mutation is a key search operator which generates new solutions from the current ones. A new mutation operator based on Cauchy random numbers is proposed and tested on a suite of 23 functions in this paper. The new EP with Cauchy mutation significantly outperforms the classical EP (CEP), which uses Gaussian mutation, on a number of multimodal functions with many local minima while being comparable to CEP for unimodal and multimodal functions with only a few local minima. The new EP is denoted as "fast EP" (FEP) in this paper.

Extensive empirical studies of both FEP and CEP have been carried out to evaluate the relative strength and weakness of FEP and CEP for different problems. The results show that Cauchy mutation is an efficient search operator for a large class of multimodal function optimization problems. FEP's performance can be expected to improve further since all the parameters used in the FEP were set equivalently to those used in CEP.

To explain why Cauchy mutation performs better than Gaussian mutation for most benchmark problems used here, theoretical analysis has been carried out to show the importance of the neighborhood size and search step size in EP. It is shown that Cauchy mutation performs better because of its higher probability of making longer jumps. Although the idea behind FEP appears to be simple and straightforward (the larger the search step size, the faster the algorithm gets to the global optimum), no theoretical result has been provided so far to answer the question why this is the case and how fast it might be. In addition, a large step size may not be beneficial at all if the current search point is already very close to the global optimum. This paper shows for the first time the relationship between the distance to the global optimum and the search step size, and the relationship between the search step size and the probability of finding a near (global) optimum. Based on such analyses, an improved FEP has been proposed and tested empirically.

The rest of this paper is organized as follows. Section II describes the global minimization problem considered in this 
paper and the CEP used to solve it. The CEP algorithm given follows suggestions from Fogel [3], [6] and Bäck and Schwefel [7]. Section III describes the FEP and its implementation. Section IV gives the 23 functions used in our studies. Section $\mathrm{V}$ presents the experimental results and discussions on FEP and CEP. Section VI investigates FEP with different scale parameters for its Cauchy mutation. Section VII analyzes FEP and CEP and explains the performance difference between FEP and CEP in depth. Based on such analyses, an improved FEP (IFEP) is proposed and tested in Section VIII. Finally, Section IX concludes with some remarks and future research directions.

\section{FUNCTION OPTIMIZATION BY Classical Evolutionary Programming}

A global minimization problem can be formalized as a pair $(S, f)$, where $S \subseteq R^{n}$ is a bounded set on $R^{n}$ and $f: S \mapsto R$ is an $n$-dimensional real-valued function. The problem is to find a point $\mathrm{x}_{\min } \in S$ such that $f\left(\mathrm{x}_{\min }\right)$ is a global minimum on $S$. More specifically, it is required to find an $\mathbf{x}_{\min } \in S$ such that

$$
\forall \mathrm{x} \in S: f\left(\mathrm{x}_{\min }\right) \leq f(\mathrm{x})
$$

where $f$ does not need to be continuous but it must be bounded. This paper only considers unconstrained function optimization.

Fogel [3], [8] and Bäck and Schwefel [7] have indicated that CEP with self-adaptive mutation usually performs better than CEP without self-adaptive mutation for the functions they tested. Hence the CEP with self-adaptive mutation will be investigated in this paper. According to the description by Bäck and Schwefel [7], the CEP is implemented as follows in this study. ${ }^{1}$

1) Generate the initial population of $\mu$ individuals, and set $k=1$. Each individual is taken as a pair of realvalued vectors, $\left(\mathbf{x}_{i}, \eta_{i}\right), \forall i \in\{1, \ldots, \mu\}$, where $\mathbf{x}_{i}$ 's are objective variables and $\eta_{i}$ 's are standard deviations for Gaussian mutations (also known as strategy parameters in self-adaptive evolutionary algorithms).

2) Evaluate the fitness score for each individual $\left(\mathbf{x}_{i}, \eta_{i}\right)$, $\forall i \in\{1, \ldots, \mu\}$, of the population based on the objective function, $f\left(\mathbf{x}_{i}\right)$.

3) Each parent $\left(\mathrm{x}_{i}, \eta_{i}\right), i=1, \ldots, \mu$, creates a single offspring $\left(\mathrm{x}_{i}^{\prime}, \eta_{i}^{\prime}\right)$ by: for $j=1, \ldots, n$

$$
\begin{aligned}
x_{i}^{\prime}(j) & =x_{i}(j)+\eta_{i}(j) N_{j}(0,1) \\
\eta_{i}^{\prime}(j) & =\eta_{i}(j) \exp \left(\tau^{\prime} N(0,1)+\tau N_{j}(0,1)\right)
\end{aligned}
$$

where $x_{i}(j), x_{i}^{\prime}(j), \eta_{i}(j)$ and $\eta_{i}^{\prime}(j)$ denote the $j$-th component of the vectors $\mathbf{x}_{i}, \mathrm{x}_{i}^{\prime}, \eta_{i}$ and $\eta_{i}^{\prime}$, respectively. $N(0,1)$ denotes a normally distributed one-dimensional random number with mean zero and standard deviation one. $N_{j}(0,1)$ indicates that the random number is generated anew for each value of $j$. The factors $\tau$ and $\tau^{\prime}$ are commonly set to $(\sqrt{2 \sqrt{n}})^{-1}$ and $(\sqrt{2 n})^{-1}$ [7], [6].

\footnotetext{
${ }^{1}$ A recent study by Gehlhaar and Fogel [9] showed that swapping the order of (1) and (2) may improve CEP's performance.
}

4) Calculate the fitness of each offspring $\left(\mathrm{x}_{i}^{\prime}, \eta_{i}^{\prime}\right), \forall i \in$ $\{1, \ldots, \mu\}$.

5) Conduct pairwise comparison over the union of parents $\left(\mathbf{x}_{i}, \eta_{i}\right)$ and offspring $\left(\mathbf{x}_{i}^{\prime}, \eta_{i}^{\prime}\right), \forall i \in\{1, \ldots, \mu\}$. For each individual, $q$ opponents are chosen uniformly at random from all the parents and offspring. For each comparison, if the individual's fitness is no smaller than the opponent's, it receives a "win."

6) Select the $\mu$ individuals out of $\left(\mathrm{x}_{i}, \eta_{i}\right)$ and $\left(\mathrm{x}_{i}^{\prime}, \eta_{i}^{\prime}\right)$, $\forall i \in\{1, \ldots, \mu\}$, that have the most wins to be parents of the next generation.

7) Stop if the halting criterion is satisfied; otherwise, $k=$ $k+1$ and go to Step 3 .

\section{Fast Evolutionary Programming}

The one-dimensional Cauchy density function centered at the origin is defined by

$$
f_{t}(x)=\frac{1}{\pi} \frac{t}{t^{2}+x^{2}}, \quad-\infty<x<\infty
$$

where $t>0$ is a scale parameter [10,p. 51]. The corresponding distribution function is

$$
F_{t}(x)=\frac{1}{2}+\frac{1}{\pi} \arctan \left(\frac{x}{t}\right) .
$$

The shape of $f_{t}(x)$ resembles that of the Gaussian density function but approaches the axis so slowly that an expectation does not exist. As a result, the variance of the Cauchy distribution is infinite. Fig. 1 shows the difference between Cauchy and Gaussian functions by plotting them in the same scale.

The FEP studied in this paper is exactly the same as the CEP described in Section II except for (1) which is replaced by the following [11]

$$
x_{i}^{\prime}(j)=x_{i}(j)+\eta_{i}(j) \delta_{j}
$$

where $\delta_{j}$ is a Cauchy random variable with the scale parameter $t=1$ and is generated anew for each value of $j$. It is worth indicating that we leave (2) unchanged in FEP to keep our modification of CEP to a minimum. It is also easy to investigate the impact of the Cauchy mutation on EP when other parameters are kept the same.

It is clear from Fig. 1 that Cauchy mutation is more likely to generate an offspring further away from its parent than Gaussian mutation due to its long flat tails. It is expected to have a higher probability of escaping from a local optimum or moving away from a plateau, especially when the "basin of attraction" of the local optimum or the plateau is large relative to the mean step size. On the other hand, the smaller hill around the center in Fig. 1 indicates that Cauchy mutation spends less time in exploiting the local neighborhood and thus has a weaker fine-tuning ability than Gaussian mutation in small to mid-range regions. Our empirical results support the above intuition. 


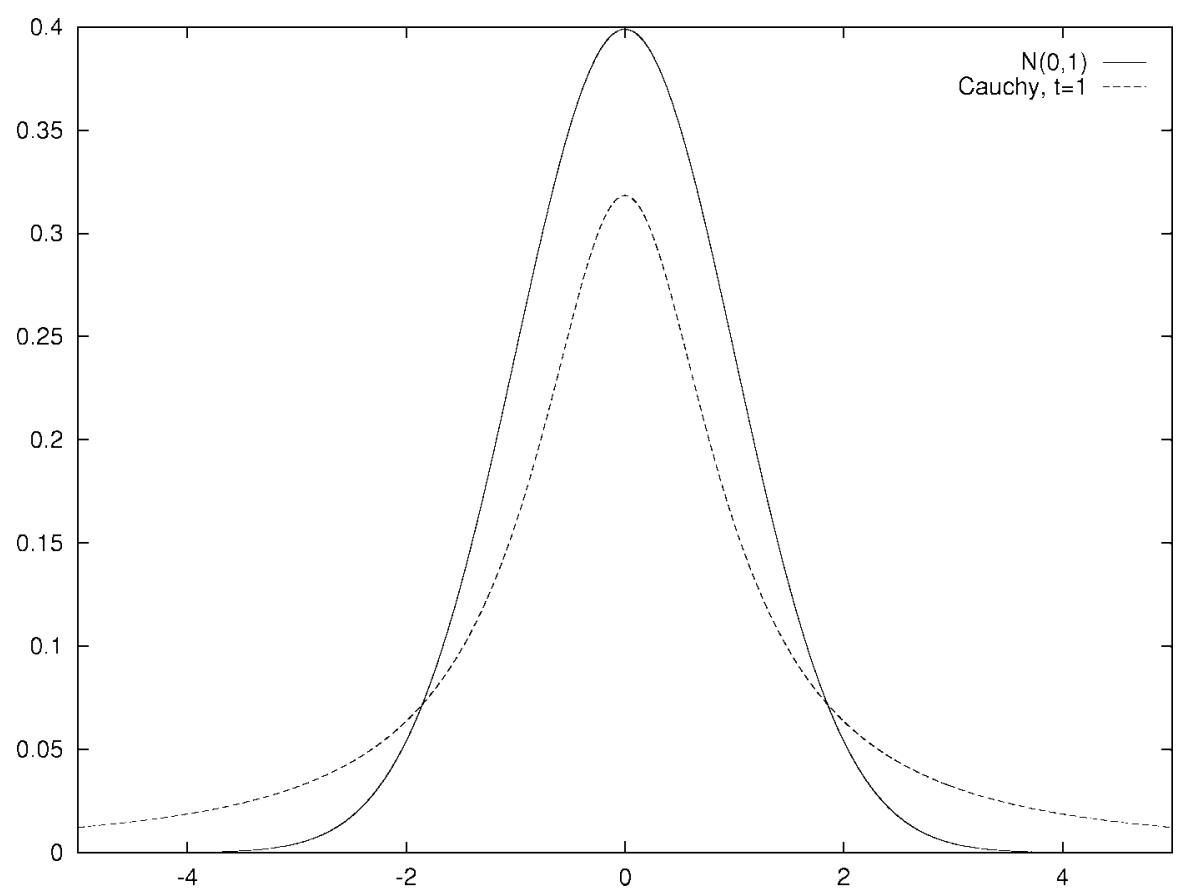

Fig. 1. Comparison between Cauchy and Gaussian density functions.

\section{BENCHMARK FUNCTIONS}

Twenty-three benchmark functions [2], [7], [12], [13] were used in our experimental studies. This number is larger than that offered in many other empirical study papers. This is necessary, however, since the aim here is not to show FEP is better or worse than CEP, but to find out when FEP is better (or worse) than CEP and why. Wolpert and Macready [14], [15] have shown that under certain assumptions no single search algorithm is best on average for all problems. If the number of test problems is small, it would be very difficult to make a generalized conclusion. Using too small a test set also has the potential risk that the algorithm is biased (optimized) toward the chosen problems, while such bias might not be useful for other problems of interest.

The 23 benchmark functions are given in Table I. A more detailed description of each function is given in the Appendix. Functions $f_{1}-f_{13}$ are high-dimensional problems. Functions $f_{1}-f_{5}$ are unimodal. Function $f_{6}$ is the step function, which has one minimum and is discontinuous. Function $f_{7}$ is a noisy quartic function, where $\operatorname{random}[0,1)$ is a uniformly distributed random variable in $[0,1)$. Functions $f_{8}-f_{13}$ are multimodal functions where the number of local minima increases exponentially with the problem dimension [12], [13]. They appear to be the most difficult class of problems for many optimization algorithms (including EP). Functions $f_{14}-f_{23}$ are low-dimensional functions which have only a few local minima [12]. For unimodal functions, the convergence rates of FEP and CEP are more interesting than the final results of optimization as there are other methods which are specifically designed to optimize unimodal functions. For multimodal functions, the final results are much more important since they reflect an algorithm's ability of escaping from poor local optima and locating a good near-global optimum.

\section{EXPERIMENTAL STUDIES}

\section{A. Experimental Setup}

In all experiments, the same self-adaptive method [i.e., (2)], the same population size $\mu=100$, the same tournament size $q=10$ for selection, the same initial $\eta=3.0$, and the same initial population were used for both CEP and FEP. These parameters follow the suggestions from Bäck and Schwefel [7] and Fogel [2]. The initial population was generated uniformly at random in the range as specified in Table $\mathrm{I}$.

\section{B. Unimodal Functions}

The first set of experiments was aimed to compare the convergence rate of CEP and FEP for functions $f_{1}-f_{7}$. The average results of 50 independent runs are summarized in Table II. Figs. 2 and 3 show the progress of the mean best solutions and the mean of average values of population found by CEP and FEP over 50 runs for $f_{1}-f_{7}$. It is apparent that FEP performs better than CEP in terms of convergence rate although CEP's final results were better than FEP's for functions $f_{1}$ and $f_{2}$. Function $f_{1}$ is the simple sphere model studied by many researchers. CEP's and FEP's behaviors on $f_{1}$ are quite illuminating. In the beginning, FEP displays a faster convergence rate than CEP due to its better global search ability. It quickly approaches the neighborhood of the global optimum and reaches approximately 0.001 in around 1200 generations, while CEP can only reach approximately 0.1. After that, FEP's convergence rate reduces substantially, while CEP maintains a nearly constant convergence rate throughout the evolution. Finally, CEP overtakes FEP at around generation 1450. As indicated in Section III and in Fig. 1, FEP is weaker than CEP in fine tuning. Such weakness slows down its convergence considerably in the neighborhood 
TABLE I

The 23 Benchmark Functions Used in Our Experimental Study, Where $n$ Is the Dimension of the Function, $f_{\text {min }}$ Is the Minimum Value of the Function, and $S \subseteq R^{n}$. A Detailed Description of All Functions Is Given in the Appendix

\begin{tabular}{|c|c|c|c|}
\hline Test function & $n$ & $S$ & $f_{\min }$ \\
\hline$f_{1}(x)=\sum_{i=1}^{n} x_{i}^{2}$ & 30 & {$[-100,100]^{n}$} & 0 \\
\hline$f_{2}(x)=\sum_{i=1}^{n}\left|x_{i}\right|+\prod_{i=1}^{n}\left|x_{i}\right|$ & 30 & {$[-10,10]^{n}$} & 0 \\
\hline$f_{3}(x)=\sum_{i=1}^{n}\left(\sum_{j=1}^{i} x_{j}\right)^{2}$ & 30 & {$[-100,100]^{n}$} & 0 \\
\hline$f_{4}(x)=\max _{i}\left\{\left|x_{i}\right|, 1 \leq i \leq n\right\}$ & 30 & {$[-100,100]^{n}$} & 0 \\
\hline$f_{5}(x)=\sum_{i=1}^{n-1}\left[100\left(x_{i+1}-x_{i}^{2}\right)^{2}+\left(x_{i}-1\right)^{2}\right]$ & 30 & {$[-30,30]^{n}$} & 0 \\
\hline$f_{6}(x)=\sum_{i=1}^{n}\left(\left\lfloor x_{i}+0.5\right\rfloor\right)^{2}$ & 30 & {$[-100,100]^{n}$} & 0 \\
\hline$f_{7}(x)=\sum_{i=1}^{n=1} i x_{i}^{4}+\operatorname{random}[0,1)$ & 30 & {$[-1.28,1.28]^{n}$} & 0 \\
\hline$f_{8}(x)=\sum_{i=1}^{n-1}-x_{i} \sin \left(\sqrt{\left|x_{i}\right|}\right)$ & 30 & {$[-500,500]^{n_{2}}$} & -12569.5 \\
\hline$\left.f_{9}(x)=\sum_{i=1}^{n}\left[x_{i}^{2}-10 \cos \left(2 \pi x_{i}\right)+10\right)\right]$ & 30 & {$[-5.12,5.12]^{n}$} & 0 \\
\hline $\begin{aligned} f_{10}(x)= & -20 \exp \left(-0.2 \sqrt{\frac{1}{n} \sum_{i=1}^{n} x_{i}^{2}}\right)-\exp \left(\frac{1}{n} \sum_{i=1}^{n} \cos 2 \pi x_{i}\right) \\
& +20+e\end{aligned}$ & 30 & {$[-32,32]^{n}$} & 0 \\
\hline$f_{11}(x)=\frac{1}{4000} \sum_{i=1}^{n} x_{i}^{2}-\prod_{i=1}^{n} \cos \left(\frac{x_{i}}{\sqrt{i}}\right)+1$ & 30 & {$[-600,600]^{n}$} & 0 \\
\hline $\begin{aligned} f_{12}(x)= & \frac{\pi}{n}\left\{10 \sin ^{2}\left(\pi y_{i}\right)+\sum_{i=1}^{n-1}\left(y_{i}-1\right)^{2}\left[1+10 \sin ^{2}\left(\pi y_{i+1}\right)\right]\right. \\
& \left.+\left(y_{n}-1\right)^{2}\right\}+\sum_{i=1}^{n} u\left(x_{i}, 10,100,4\right) \\
& y_{i}=1+\frac{1}{4}\left(x_{i}+1\right)\end{aligned}$ & 30 & {$[-50,50]^{n}$} & 0 \\
\hline$u\left(x_{i}, a, k, m\right)= \begin{cases}k\left(x_{i}-a\right)^{m}, & x_{i}>a \\
0, & -a \leq x_{i} \leq a \\
k\left(-x_{i}-a\right)^{m}, & x_{i}<-a\end{cases}$ & & & \\
\hline $\begin{aligned} f_{13}(x)= & 0.1\left\{\sin ^{2}\left(3 \pi x_{1}\right)+\sum_{i=1}^{n-1}\left(x_{i}-1\right)^{2}\left[1+\sin ^{2}\left(3 \pi x_{i+1}\right)\right]\right. \\
& \left.+\left(x_{n}-1\right)\left[1+\sin ^{2}\left(2 \pi x_{n}\right)\right]\right\}+\sum_{i=1}^{n} u\left(x_{i}, 5,100,4\right)\end{aligned}$ & 30 & {$[-50,50]^{n}$} & 0 \\
\hline$f_{14}(x)=\left[\frac{1}{500}+\sum_{j=1}^{25} \frac{1}{j+\sum_{i=1}^{2}\left(x_{i}-a_{i j}\right)^{6}}\right]$ & 2 & {$[-65.536,65.536]^{n}$} & 1 \\
\hline$f_{15}(x)=\sum_{i=1}^{11}\left[a_{i}-\frac{x_{1}\left(b_{i}^{2}+b_{i} x_{2}\right)}{b_{i}^{2}+b_{i} x_{3}+x_{4}}\right]^{2}$ & 4 & {$[-5,5]^{n}$} & 0.0003075 \\
\hline$f_{16}(x)=4 x_{1}^{2}-2.1 x_{1}^{4}+\frac{1}{3} x_{1}^{6}+x_{1} x_{2}-4 x_{2}^{2}+4 x_{2}^{4}$ & 2 & {$[-5,5]^{n}$} & -1.0316285 \\
\hline $\begin{aligned} f_{17}(x)= & \left(x_{2}-\frac{5.1}{4 \pi^{2}} x_{1}^{2}+\frac{5}{\pi} x_{1}-6\right)^{2}+10\left(1-\frac{1}{8 \pi}\right) \cos x_{1}+10 \\
f_{18}(x)= & {\left[1+\left(x_{1}+x_{2}+1\right)^{2}\left(19-14 x_{1}+3 x_{1}^{2}-14 x_{2}\right.\right.} \\
& \left.\left.+6 x_{1} x_{2}+3 x_{2}^{2}\right)\right] \times\left[30+\left(2 x_{1}-3 x_{2}\right)^{2}\left(18-32 x_{1}\right.\right. \\
& \left.\left.+12 x_{1}^{2}+48 x_{2}-36 x_{1} x_{2}+27 x_{2}^{2}\right)\right]\end{aligned}$ & $\begin{array}{l}2 \\
2\end{array}$ & $\begin{array}{c}{[-5,10] \times[0,15]} \\
{[-2,2]^{n}}\end{array}$ & $\begin{array}{c}0.398 \\
3\end{array}$ \\
\hline$f_{19}(x)=-\sum_{i=1}^{4} c_{i} \exp \left[-\sum_{j=1}^{4} a_{i j}\left(x_{j}-p_{i j}\right)^{2}\right]$ & 4 & {$[0,1]^{n}$} & -3.86 \\
\hline$f_{20}(x)=-\sum_{i=1}^{4} c_{i} \exp \left[-\sum_{j=1}^{6} a_{i j}\left(x_{j}-p_{i j}\right)^{2}\right]$ & 6 & {$[0,1]^{n}$} & -3.32 \\
\hline$f_{21}(x)=-\sum_{i=1}^{5}\left[\left(x-a_{i}\right)\left(x-a_{i}\right)^{T}+c_{i}\right]^{-1}$ & 4 & {$[0,10]^{n}$} & -10 \\
\hline$f_{22}(x)=-\sum_{i=1}^{7}\left[\left(x-a_{i}\right)\left(x-a_{i}\right)^{T}+c_{i}\right]^{-1}$ & 4 & {$[0,10]^{n}$} & -10 \\
\hline$f_{23}(x)=-\sum_{i=1}^{10}\left[\left(x-a_{i}\right)\left(x-a_{i}\right)^{T}+c_{i}\right]^{-1}$ & 4 & {$[0,10]^{n_{L}}$} & -10 \\
\hline
\end{tabular}

TABLE II

Comparison Between CEP and FeP on $f_{1}-f_{7}$. All Results Have Been Averaged over 50 Runs, Where "Mean Best" Indicates the Mean Best Function Values Found in the Last Generation, and "Std Dev" Stands for the Standard Deviation

\begin{tabular}{|c|c|c|c|c|c|c|}
\hline \multirow[t]{2}{*}{ Function } & \multirow{2}{*}{$\begin{array}{l}\text { Number of } \\
\text { Generations }\end{array}$} & \multicolumn{2}{|c|}{ FEP } & \multicolumn{2}{|c|}{ CEP } & \multirow{2}{*}{$\begin{array}{c}\mathrm{FEP}-\mathrm{CEP} \\
t \text {-test }\end{array}$} \\
\hline & & Mean Best & Std Dev & Mean Best & Std Dev & \\
\hline$f_{1}$ & 1500 & $5.7 \times 10^{-4}$ & $1 . \overline{3 \times 10^{-4}}$ & $2.2 \times 10^{-4}$ & $5.9 \times 10^{-4}$ & $4.06^{\dagger}$ \\
\hline$f_{2}$ & 2000 & $8.1 \times 10^{-3}$ & $7.7 \times 10^{-4}$ & $2.6 \times 10^{-3}$ & $1.7 \times 10^{-4}$ & $49.83^{\dagger}$ \\
\hline$f_{3}$ & 5000 & $1.6 \times 10^{-2}$ & $1.4 \times 10^{-2}$ & $5.0 \times 10^{-2}$ & $6.6 \times 10^{-2}$ & $-3.79^{\dagger}$ \\
\hline$f_{4}$ & 5000 & 0.3 & 0.5 & 2.0 & 1.2 & $-8.25^{\dagger}$ \\
\hline$f_{5}$ & 20000 & 5.06 & 5.87 & 6.17 & 13.61 & -0.52 \\
\hline$f_{6}$ & 1500 & 0 & 0 & 577.76 & 1125.76 & $-3.67^{\dagger}$ \\
\hline$f_{7}$ & 3000 & $7.6 \times 10^{-3}$ & $2.6 \times 10^{-3}$ & $1.8 \times 10^{-2}$ & $6.4 \times 10^{-3}$ & $-10.72^{\dagger}$ \\
\hline
\end{tabular}

†The value of $t$ with 49 degrees of freedom is significant at $\alpha=0.05$ by a two-tailed test.

of the global optimum. CEP is capable of maintaining its nearly constant convergence rate because its search is much more localized than FEP. The different behavior of CEP and FEP on $f_{1}$ suggests that CEP is better at fine-grained search while FEP is better at coarse-grained search.

The largest difference in performance between CEP and FEP occurs with function $f_{6}$, the step function, which is characterized by plateaus and discontinuity. CEP performs poorly on the step function because it mainly searches in a relatively small local neighborhood. All the points within the local neighborhood will have the same fitness value except for a few boundaries between plateaus. Hence it is very difficult for CEP to move from one plateau to a lower one. On the other hand, FEP has a much higher probability of 
f 1 (Sphere Model)
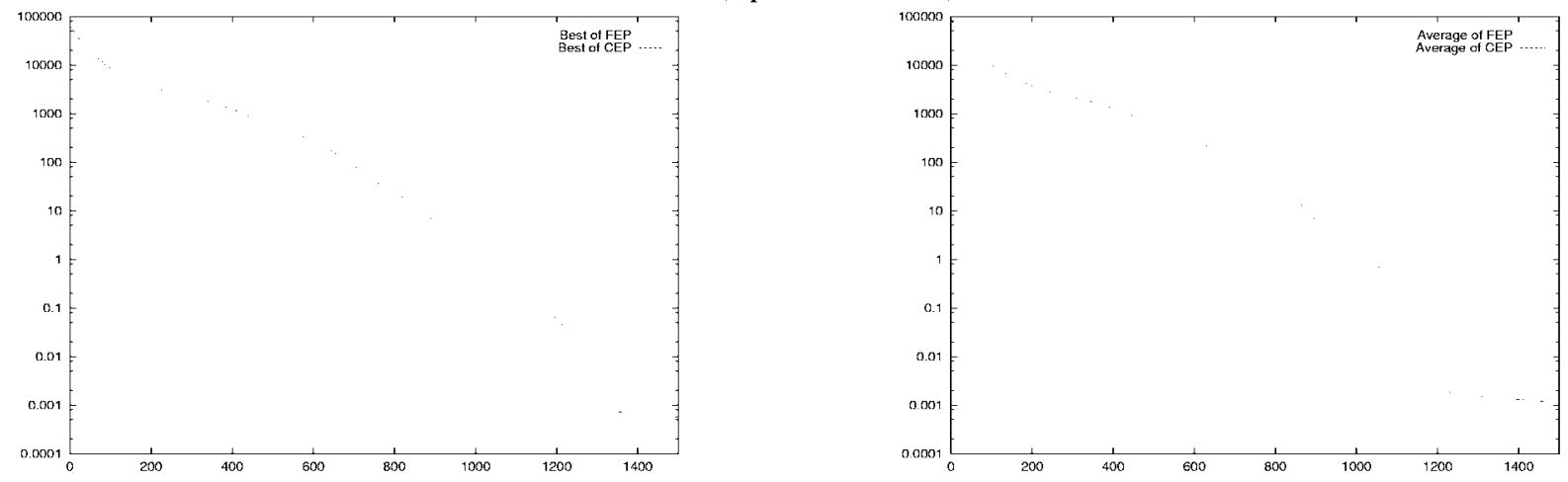

$f_{2}$ (Schwefel's Problem 2.22)
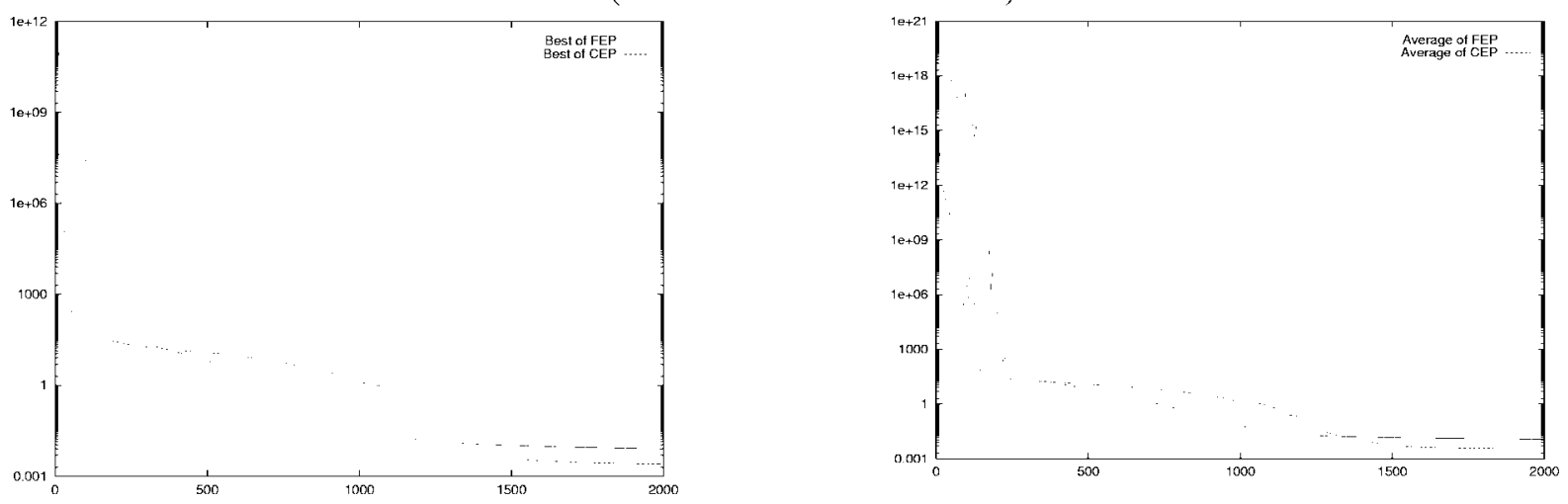

\section{$\mathrm{f}_{3}$ (Schwefel's Problem 1.2)}
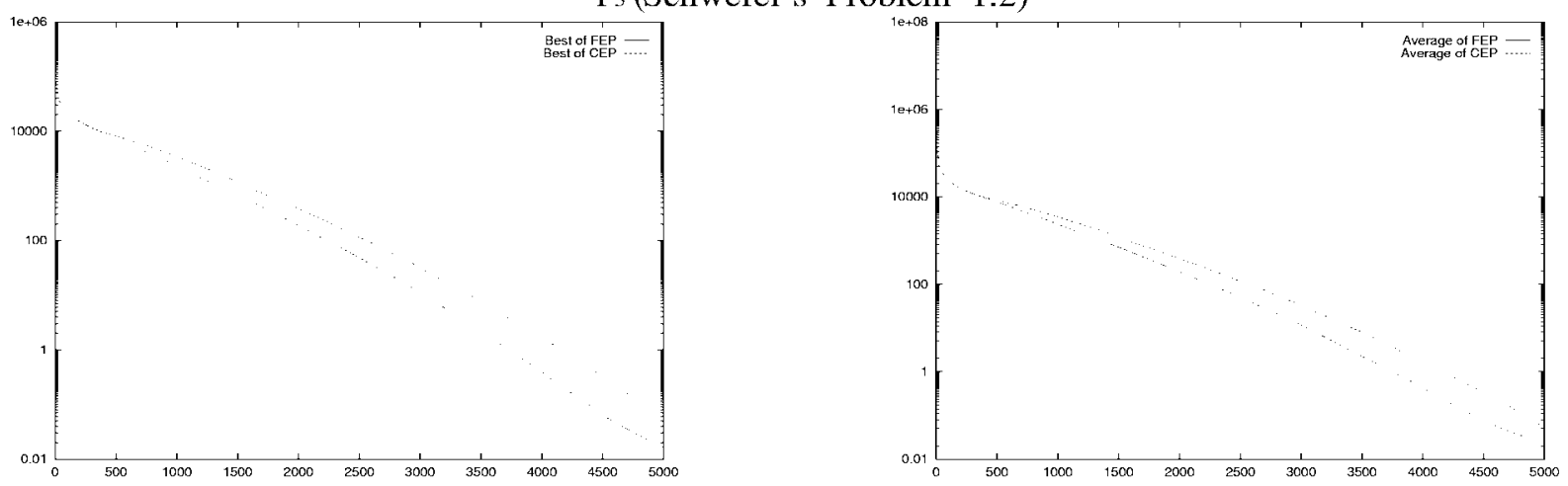

$f_{4}$ (Schwefel's Problem 2.21)

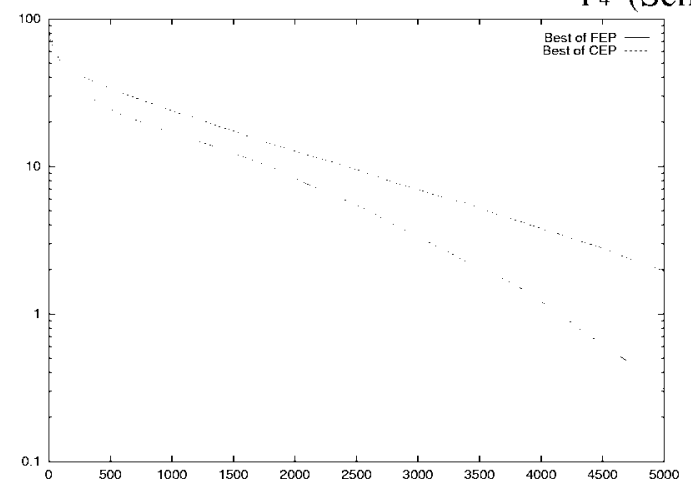

(a)

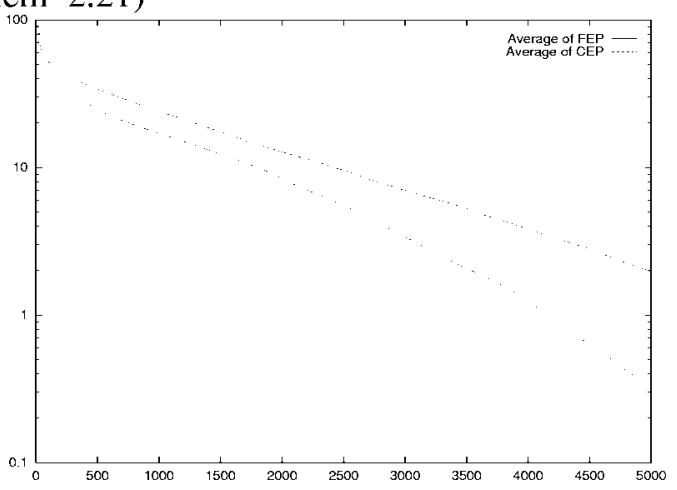

(b)

Fig. 2. Comparison between CEP and FEP on $f_{1}-f_{4}$. The vertical axis is the function value, and the horizontal axis is the number of generations. The solid lines indicate the results of FEP. The dotted lines indicate the results of CEP. (a) shows the best results, and (b) shows the average results. Both were averaged over 50 runs. 
$f_{5}$ (Generalized Rosenbrock's Function)
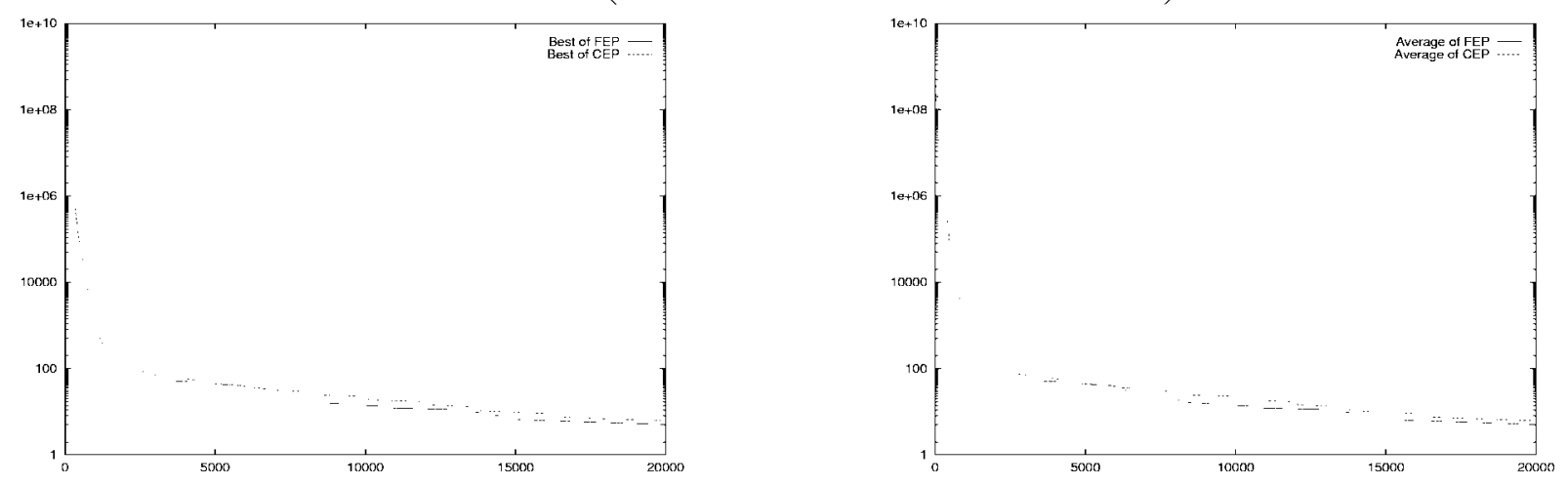

$f_{6}$ (Step Function)
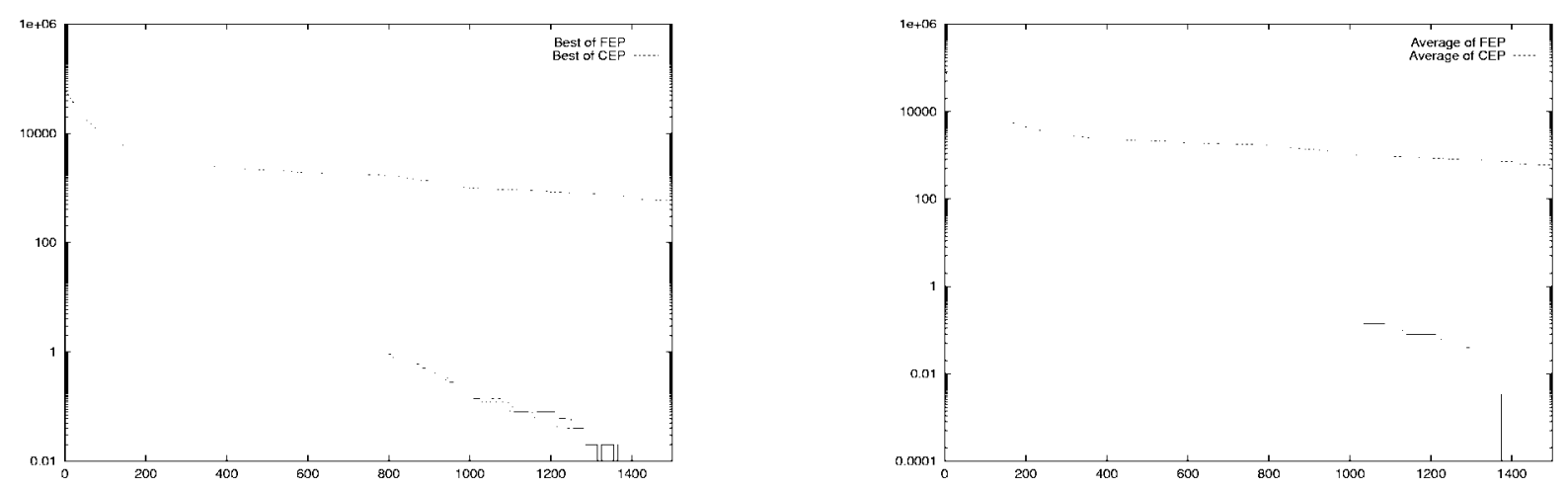

$f_{7}$ (Quartic Function with Noise)

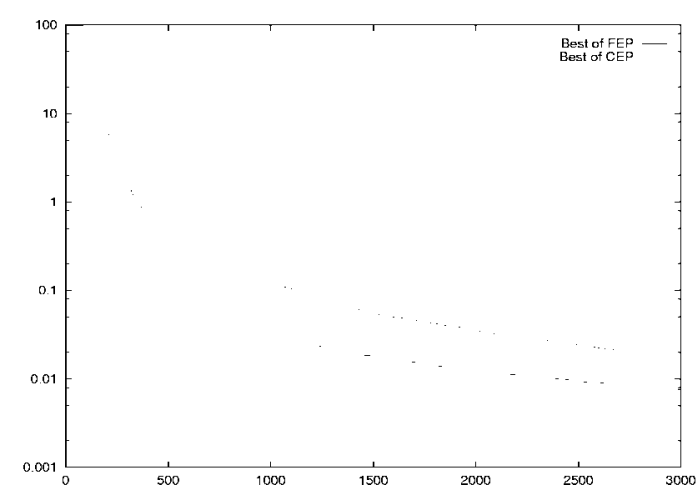

(a)

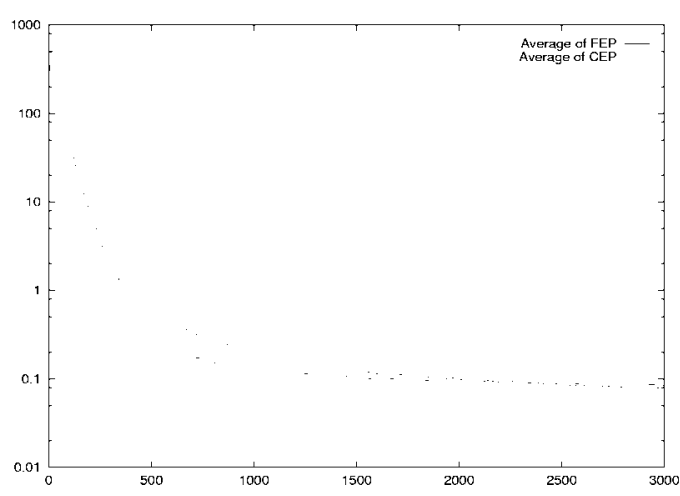

(b)

Fig. 3. Comparison between CEP and FEP on $f_{5}-f_{7}$. The vertical axis is the function value, and the horizontal axis is the number of generations. The solid lines indicate the results of FEP. The dotted lines indicate the results of CEP. (a) shows the best results, and (b) shows average results. Both were averaged over 50 runs.

generating long jumps than CEP. Such long jumps enable FEP to move from one plateau to a lower one with relative ease. The rapid convergence of FEP shown in Fig. 3 supports our explanations.

\section{Multimodal Functions}

1) Multimodal Functions with Many Local Minima: Multimodal functions having many local minima are often regarded as being difficult to optimize. $f_{8}-f_{13}$ are such functions where the number of local minima increases exponentially as the dimension of the function increases. Fig. 4 shows the two-dimensional version of $f_{8}$.

The dimensions of $f_{8}-f_{13}$ were all set to 30 in our experiments. Table III summarizes the final results of CEP and FEP. It is obvious that FEP performs significantly better than CEP consistently for these functions. CEP appeared to become trapped in a poor local optimum and unable to escape from it due to its smaller probability of making long jumps. According to the figures we plotted to observe the evolutionary process, CEP fell into a poor local optimum quite early in a run while 


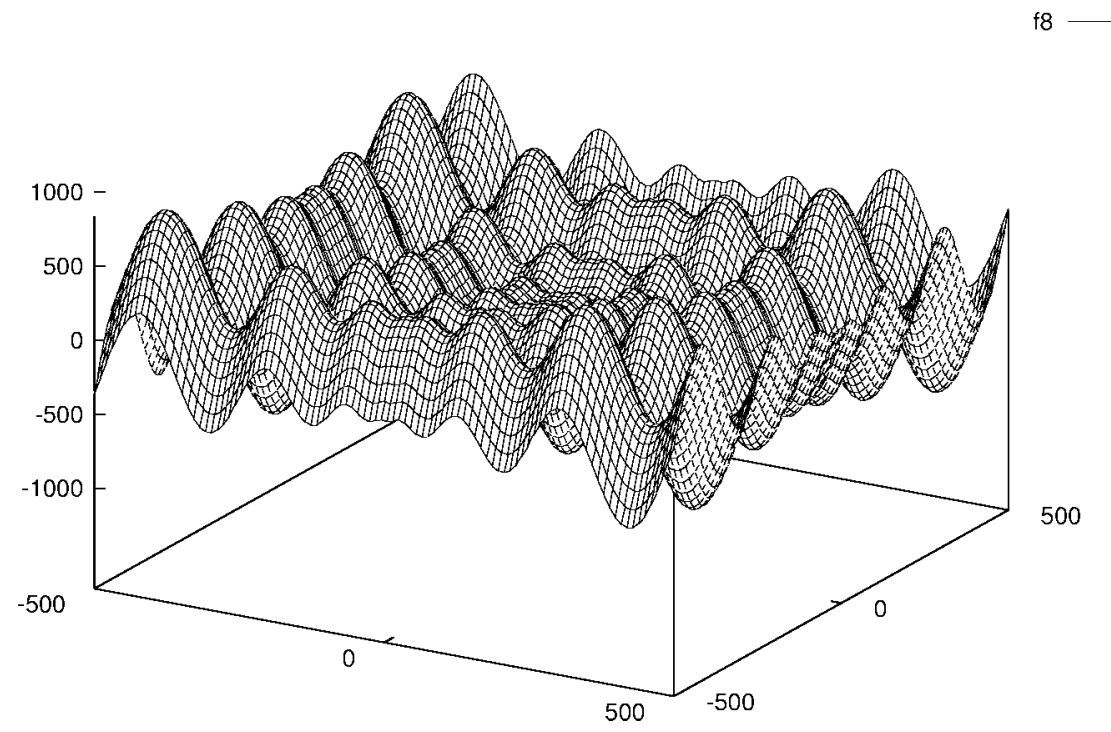

Fig. 4. The two-dimensional version of $f_{8}$.

TABLE III

Comparison Between CEP and FeP on $f_{8}-f_{13}$. The Results Are Averaged over 50 Runs, Where "Mean Best" Indicates the Mean Best Function Values Found in the Last Generation and "Std Dev" Stands for the Standard Deviation

\begin{tabular}{|c|c|c|c|c|c|c|}
\hline \multirow[t]{2}{*}{ Function } & \multirow{2}{*}{$\begin{array}{l}\text { Number of } \\
\text { Generations }\end{array}$} & \multicolumn{2}{|c|}{ FEP } & \multicolumn{2}{|c|}{ CEP } & \multirow{2}{*}{$\begin{array}{c}\mathrm{FEP}-\mathrm{CEP} \\
t \text {-test }\end{array}$} \\
\hline & & Mean Best & Std Dev & Mean Best & Std Dev & \\
\hline$f_{8}$ & 9000 & -12554.5 & 52.6 & -7917.1 & 634.5 & $-51.39^{\dagger}$ \\
\hline$f_{9}$ & 5000 & $4.6 \times 10^{-2}$ & $1.2 \times 10^{-2}$ & 89.0 & 23.1 & $-27.25^{\dagger}$ \\
\hline$f_{10}$ & 1500 & $1.8 \times 10^{-2}$ & $2.1 \times 10^{-3}$ & 9.2 & 2.8 & $-23.33^{\dagger}$ \\
\hline$f_{11}$ & 2000 & $1.6 \times 10^{-2}$ & $2.2 \times 10^{-2}$ & $8.6 \times 10^{-2}$ & 0.12 & $-4.28^{\dagger}$ \\
\hline$f_{12}$ & 1500 & $9.2 \times 10^{-6}$ & $3.6 \times 10^{-6}$ & 1.76 & 2.4 & $-5.29^{\dagger}$ \\
\hline$f_{13}$ & 1500 & $1.6 \times 10^{-4}$ & $7.3 \times 10^{-5}$ & 1.4 & 3.7 & $-2.76^{\dagger}$ \\
\hline
\end{tabular}

tThe value of $t$ with 49 degrees of freedom is significant at $\alpha=0.05$ by a two-tailed test.

TABLE IV

Comparison Between CEP and FEP on $f_{14}-f_{23}$. The Results Are Averaged over 50 Runs, Where "Mean Best" Indicates the Mean Best Function Values Found in the Last Generation and "Std Dev" Stands for the Standard Deviation

\begin{tabular}{llccccc}
\hline Function & Number of & \multicolumn{2}{c}{ FEP } & \multicolumn{2}{c}{ CEP } & FEP-CEP \\
& Generations & Mean Best & Std Dev & Mean Best & Std Dev & $t$-test \\
\hline$f_{14}$ & 100 & 1.22 & 0.56 & 1.66 & 1.19 & $-2.21^{\dagger}$ \\
$f_{15}$ & 4000 & $5.0 \times 10^{-4}$ & $3.2 \times 10^{-4}$ & $4.7 \times 10^{-4}$ & $3.0 \times 10^{-4}$ & 0.49 \\
$f_{16}$ & 100 & -1.03 & $4.9 \times 10^{-7}$ & -1.03 & $4.9 \times 10^{-7}$ & 0.0 \\
$f_{17}$ & 100 & 0.398 & $1.5 \times 10^{-7}$ & 0.398 & $1.5 \times 10^{-7}$ & 0.0 \\
$f_{18}$ & 100 & 3.02 & 0.11 & 3.0 & 0 & 1.0 \\
$f_{19}$ & 100 & -3.86 & $1.4 \times 10^{-5}$ & -3.86 & $1.4 \times 10^{-2}$ & -1.0 \\
$f_{20}$ & 200 & -3.27 & $5.9 \times 10^{-2}$ & -3.28 & $5.8 \times 10^{-2}$ & 0.45 \\
$f_{21}$ & 100 & -5.52 & 1.59 & -6.86 & 2.67 & $3.56^{\dagger}$ \\
$f_{22}$ & 100 & -5.52 & 2.12 & -8.27 & 2.95 & $5.44^{\dagger}$ \\
$f_{23}$ & 100 & -6.57 & 3.14 & -9.10 & 2.92 & $4.24^{\dagger}$ \\
\hline
\end{tabular}

tThe value of $t$ with 49 degrees of freedom is significant at $\alpha=0.05$ by a two-tailed test.

FEP was able to improve its solution steadily for a long time. FEP appeared to converge at least at a linear rate with respect to the number of generations. An exponential convergence rate was observed for some problems.

2) Multimodal Functions with Only a Few Local Minima: To evaluate FEP more fully, additional multimodal benchmark functions were also included in our experiments, i.e., $f_{14}-f_{23}$, where the number of local minima for each function and the dimension of the function are small. Table IV summarizes the results averaged over 50 runs.

Interestingly, quite different results have been observed for functions $f_{14}-f_{23}$. For six (i.e., $f_{15}-f_{20}$ ) out of ten functions, no statistically significant difference was found between FEP and CEP. In fact, FEP performed exactly the same as CEP for 
TABLE V

Comparison Between CEP and FEP on $f_{8}$ To $f_{13}$ with $n=5$. The Results Are Averaged over 50 Runs, Where "Mean Best" Indicates the Mean Best Function Values Found in the Last Generation and "Std Dev" Stands for the Standard Deviation

\begin{tabular}{|c|c|c|c|c|c|c|}
\hline \multirow[t]{2}{*}{ Function } & \multirow{2}{*}{$\begin{array}{l}\text { Number of } \\
\text { Generations }\end{array}$} & \multicolumn{2}{|c|}{ FEP } & \multicolumn{2}{|c|}{$\mathrm{CEP}$} & \multirow{2}{*}{$\begin{array}{c}\mathrm{FEP}-\mathrm{CEP} \\
t \text {-test }\end{array}$} \\
\hline & & Mean Best & Std Dev & Mean Best & Std Dev & \\
\hline$f_{8}$ & 500 & -2061.74 & 58.79 & -1762.45 & 176.21 & $-11.17^{\dagger}$ \\
\hline$f_{9}$ & 400 & 0.14 & 0.40 & 4.08 & 3.08 & $-8.89^{\dagger}$ \\
\hline$f_{10}$ & 400 & $8.6 \times 10^{-4}$ & $1.8 \times 10^{-4}$ & $8.1 \times 10^{-2}$ & 0.34 & -1.67 \\
\hline$f_{11}$ & 1500 & $5.3 \times 10^{-2}$ & $4.2 \times 10^{-2}$ & 0.14 & 0.12 & $-4.64^{\dagger}$ \\
\hline$f_{12}$ & 200 & $1.5 \times 10^{-7}$ & $1.2 \times 10^{-7}$ & $2.5 \times 10^{-2}$ & 0.12 & -1.43 \\
\hline$f_{13}$ & 200 & $3.5 \times 10^{-7}$ & $1.8 \times 10^{-7}$ & $3.8 \times 10^{-3}$ & $1.4 \times 10^{-2}$ & -1.89 \\
\hline
\end{tabular}

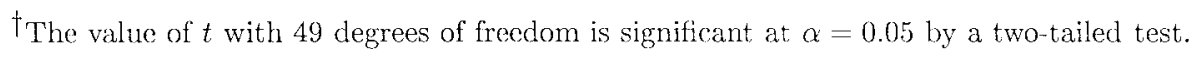

$f_{16}$ and $f_{17}$. For the four functions where there was statistically significant difference between FEP and CEP, FEP performed better for $f_{14}$, but was outperformed by CEP for $f_{21}-f_{23}$. The consistent superiority of FEP over CEP for functions $f_{8}-f_{13}$ was not observed here.

The major difference between functions $f_{8}-f_{13}$ and $f_{14}-f_{23}$ is that functions $f_{14}-f_{23}$ appear to be simpler than $f_{8}-f_{13}$ due to their low dimensionalities and a smaller number of local minima. To find out whether or not the dimensionality of functions plays a significant role in deciding FEP's and CEP's behavior, another set of experiments on the low-dimensional $(n=5)$ version of $f_{8}-f_{13}$ was carried out. The results averaged over 50 runs are given in Table V.

Very similar results to the previous ones on functions $f_{8}-f_{13}$ were obtained despite the large difference in the dimensionality of functions. FEP still outperforms CEP significantly even when the dimensionality of functions $f_{8}-f_{13}$ is low $(n=5)$. It is clear that dimensionality is not the key factor which determines the behavior of FEP and CEP. It is the shape of the function and/or the number of local minima that have a major impact on FEP's and CEP's performance.

\section{Fast EVolutionary Programming WITH DIFFERENT PARAMETERS}

The FEP investigated in the previous section used $t=1$ in its Cauchy mutation. This value was used for its simplicity. To examine the impact of different $t$ values on the performance of FEP in detail, a set of experiments have been carried out on FEP using different $t$ values for the Cauchy mutation. Seven benchmark functions from the three different groups in Table I were used in these experiments. The setup of these experiments is exactly the same as before. Table VI shows the average results over 50 independent runs of FEP for different parameters.

These results show that $t=1$ was not the optimal value for the seven benchmark problems. The optimal $t$ is problem dependent. As analyzed later in Section VII-A, the optimal $t$ depends on the distance between the current search point and the global optimum. Since the global optimum is usually unknown for real-world problems, it is extremely difficult to find the optimal $t$ for a given problem. A good approach to
TABLE VI

The Mean Best Solutions Found by FeP Using Different Scale Parameter $t$ in the Cauchy Mutation for Functions $f_{1}(1500)$, $f_{2}(2000), f_{10}(1500), f_{11}(2000), f_{21}(100), f_{22}(100)$, AND $f_{23}(100)$. the Values in “( $)$ " Indicate the Number of Generations USED iN FEP. All Results Have Been Averaged over 50 Runs

\begin{tabular}{l|c|c|c|c|c}
\hline Function & $t=0.0156$ & $t=0.0313$ & $t=0.0625$ & $t=0.1250$ & $t=0.2500$ \\
\hline$f_{1}$ & 1.0435 & 0.0599 & 0.0038 & $1.5 \times 10^{-4}$ & $6.5 \times 10^{-5}$ \\
\hline$f_{2}$ & $3.8 \times 10^{-4}$ & $3.1 \times 10^{-4}$ & $5.9 \times 10^{-4}$ & 0.0011 & 0.0021 \\
\hline$f_{10}$ & 1.5627 & 0.2858 & 0.0061 & 0.0030 & 0.0050 \\
\hline$f_{11}$ & 1.0121 & 0.2237 & 0.1093 & 0.0740 & 0.0368 \\
\hline$f_{21}$ & -6.9236 & -7.7261 & -8.0487 & -8.6473 & -8.0932 \\
\hline$f_{22}$ & -7.9211 & -8.3719 & -9.1735 & -9.8401 & -9.1587 \\
\hline$f_{23}$ & -7.8588 & -8.6935 & -9.4663 & -9.2627 & -9.8107 \\
\hline \hline Function & $t=0.5000$ & $t=0.7500$ & $t=1.0000$ & $t=1.2500$ & $t=1.5000$ \\
\hline$f_{1}$ & $1.8 \times 10^{-4}$ & $3.5 \times 10^{-4}$ & $5.7 \times 10^{-4}$ & $8.2 \times 10^{-4}$ & 0.0012 \\
\hline$f_{2}$ & 0.0041 & 0.0060 & 0.0081 & 0.0101 & 0.0120 \\
\hline$f_{10}$ & 0.0091 & 0.0136 & 0.0183 & 0.0227 & 9.1987 \\
\hline$f_{11}$ & 0.0274 & 0.0233 & 0.0161 & 0.0202 & 0.0121 \\
\hline$f_{21}$ & -6.6272 & -5.2845 & -5.5189 & -5.0095 & -5.0578 \\
\hline$f_{22}$ & -7.6829 & -6.9698 & -5.5194 & -6.1831 & -5.6476 \\
\hline$f_{23}$ & -8.5037 & -7.8622 & -6.5713 & -6.1300 & -6.5364 \\
\hline
\end{tabular}

deal with this issue is to use self-adaptation so that $t$ can gradually evolve toward its near optimum although its initial value might not be optimal.

Another approach to be explored is to mix Cauchy mutation with different $t$ values in a population so that the whole population can search both globally and locally. The percentage of each type of Cauchy mutation will be self-adaptive, rather than fixed. Hence the population may emphasize either global or local search depending on different stages in the evolutionary process.

\section{ANALYSIS OF FAST AND CLASSICAL EVOLUTIONARY PROGRAMMING}

It has been pointed out in Section III that Cauchy mutation has a higher probability of making long jumps than Gaussian mutation due to its long flat tails shown in Fig. 1. In fact, the likelihood of a Cauchy mutation generating a larger jump than a Gaussian mutation can be estimated by a simple heuristic argument. 


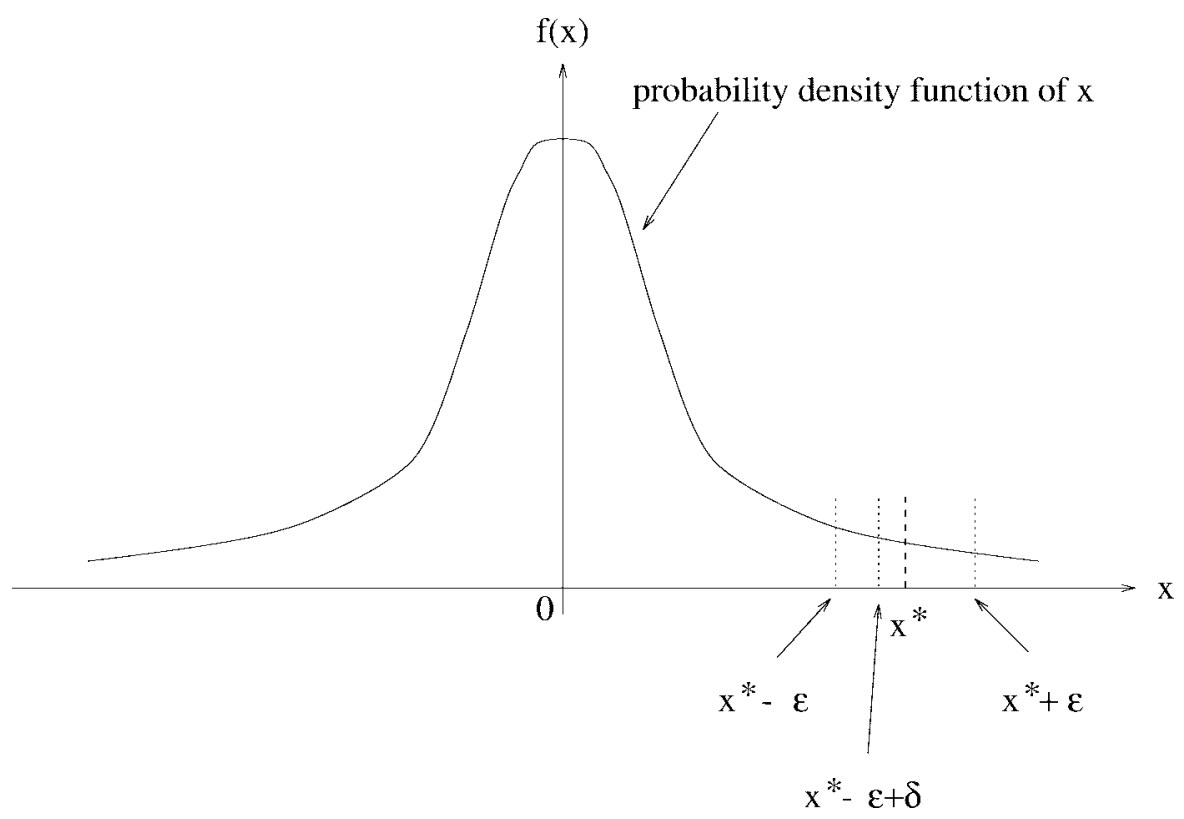

Fig. 5. Evolutionary search as neighborhood search, where $x^{*}$ is the global optimum and $\epsilon>0$ is the neighborhood size. $\delta$ is a small positive number $(0<\delta<2 \epsilon)$.

It is well known that if $N_{1}$ and $N_{2}$ are independent and identically distributed (i.i.d.) normal (Gaussian) random variates with density function

$$
f_{\text {Gaussian }}(x)=\frac{1}{\sqrt{2 \pi}} e^{-\frac{x^{2}}{2}}
$$

then $N_{1} / N_{2}$ is Cauchy distributed with density function [ 16 , p. 451]

$$
f_{\text {Cauchy }}(x)=\frac{1}{\pi\left(1+x^{2}\right)} .
$$

Given that Cauchy and Gaussian mutations follow the aforementioned distributions, Cauchy mutation will generate a larger jump than Gaussian mutation whenever $\left|N_{1} / N_{2}\right|>\left|N_{1}\right|$ (i.e., $\left|N_{2}\right|<1.0$ ). Since the probability of a Gaussian random variate smaller than 1.0 is

$$
\int_{-1}^{1} \frac{1}{\sqrt{2 \pi}} e^{-\frac{x^{2}}{2}} d x=0.68
$$

then Cauchy mutation is expected to generate a longer jump than Gaussian mutation with probability 0.68 .

The expected length of Gaussian and Cauchy jumps can be calculated as follows:

$$
\begin{aligned}
& E_{\text {Gaussian }}(x)=2 \int_{0}^{+\infty} x \frac{1}{\sqrt{2 \pi}} e^{-\frac{x^{2}}{2}} d x=\frac{2}{\sqrt{2 \pi}}=0.80 \\
& E_{\text {Cauchy }}(x)=2 \int_{0}^{+\infty} x \frac{1}{\pi\left(1+x^{2}\right)} d x=+\infty \\
& \text { (i.e., does not exist). }
\end{aligned}
$$

It is obvious that Gaussian mutation is much more localized than Cauchy mutation.
Similar results can be obtained for Gaussian distribution with expectation $m>0$ and variance $\sigma^{2}>1$ and Cauchy distribution with scale parameters $t>1$. The question now is why larger jumps would be beneficial. Is this always true?

\section{A. When Larger Jumps Are Beneficial}

Sections V-B and V-C1 have explained qualitatively why larger jumps are good at dealing with plateaus and many local optima. This section shows analytically and empirically that this is true only when the global optimum is sufficiently far away from the current search point, i.e., when the distance between the current point and the global optimum is larger than the "step size" of the mutation.

Take the Gaussian mutation in CEP as an example, which uses the following distribution with expectation zero (which implies the current search point is located at zero) and variance $\sigma^{2}$

$$
f_{G\left(0, \sigma^{2}\right)}(x)=\frac{1}{\sigma \sqrt{2 \pi}} e^{-\frac{x^{2}}{2 \sigma^{2}}}, \quad-\infty<x<+\infty .
$$

The probability of generating a point in the neighborhood of the global optimum $x^{*}$ is given by

$$
P_{G\left(0, \sigma^{2}\right)}\left(\left|x-x^{*}\right| \leq \epsilon\right)=\int_{x^{*}-\epsilon}^{x^{*}+\epsilon} f_{G\left(0, \sigma^{2}\right)}(x) d x
$$

where $\epsilon>0$ is the neighborhood size and $\sigma$ is often regarded as the step size of the Gaussian mutation. Fig. 5 illustrates the situation.

The derivative $\frac{\partial}{\partial \sigma} P_{G\left(0, \sigma^{2}\right)}\left(\left|x-x^{*}\right| \leq \epsilon\right)$ can be used to evaluate the impact of $\sigma$ on $P_{G\left(0, \sigma^{2}\right)}\left(\left|x-x^{*}\right| \leq \epsilon\right)$. According to the mean value theorem for definite integrals [17, p. 322], 
there exists a number $\delta(0<\delta<2 \epsilon)$ such that

$$
\int_{x^{*}-\epsilon}^{x^{*}+\epsilon} f_{G\left(0, \sigma^{2}\right)}(x) d x=2 \epsilon f_{G\left(0, \sigma^{2}\right)}\left(x^{*}-\epsilon+\delta\right) .
$$

Hence

$$
\begin{aligned}
\frac{\partial}{\partial \sigma} & P_{G\left(0, \sigma^{2}\right)}\left(\left|x-x^{*}\right| \leq \epsilon\right) \\
& =\frac{\partial}{\partial \sigma} \int_{x^{*}-\epsilon}^{x^{*}+\epsilon} f_{G\left(0, \sigma^{2}\right)}(x) d x \\
& =\frac{\partial}{\partial \sigma}\left(2 \epsilon f_{G\left(0, \sigma^{2}\right)}\left(x^{*}-\epsilon+\delta\right)\right) \\
& =2 \epsilon \frac{\partial}{\partial \sigma}\left(\frac{1}{\sigma \sqrt{2 \pi}} e^{-\frac{\left(x^{*}-c+\delta\right)^{2}}{2 \sigma^{2}}}\right) \\
& =2 \epsilon\left(\frac{\left(x^{*}-\epsilon+\delta\right)^{2}}{\sigma^{4} \sqrt{2 \pi}} e^{-\frac{\left(x^{*}-c+\delta\right)^{2}}{2 \sigma^{2}}}-\frac{1}{\sigma^{2} \sqrt{2 \pi}} e^{-\frac{\left(x^{*}-c+\delta\right)^{2}}{2 \sigma^{2}}}\right) \\
& =\frac{2 \epsilon}{\sigma^{2} \sqrt{2 \pi}} e^{-\frac{\left(x^{*}-c+\delta\right)^{2}}{2 \sigma^{2}}}\left(\frac{\left(x^{*}-\epsilon+\delta\right)^{2}}{\sigma^{2}}-1\right) .
\end{aligned}
$$

It is apparent from the above equation that

$$
\begin{array}{ll}
\frac{\partial}{\partial \sigma} P_{G\left(0, \sigma^{2}\right)}\left(\left|x-x^{*}\right| \leq \epsilon\right)>0 & \text { if } \sigma<\left|x^{*}-\epsilon+\delta\right| \\
\frac{\partial}{\partial \sigma} P_{G\left(0, \sigma^{2}\right)}\left(\left|x-x^{*}\right| \leq \epsilon\right)<0 & \text { if } \sigma>\left|x^{*}-\epsilon+\delta\right| .
\end{array}
$$

That is, the larger $\sigma$ is, the larger $P_{G\left(0, \sigma^{2}\right)}\left(\left|x-x^{*}\right| \leq \epsilon\right)$ will be, if $\sigma<\left|x^{*}-\epsilon+\delta\right|$. However, if $\sigma>\left|x^{*}-\epsilon+\delta\right|$, the larger $\sigma$ is, the smaller $P_{G\left(0, \sigma^{2}\right)}\left(\left|x-x^{*}\right| \leq \epsilon\right)$ will be.

Similar analysis can be carried out for Cauchy mutation in FEP. Denote the Cauchy distribution defined by (3) as $f_{C(t)}(x)$. Then we have

$$
\begin{aligned}
\frac{\partial}{\partial t} P_{C(t)}\left(\left|x-x^{*}\right| \leq \epsilon\right) & =\frac{\partial}{\partial t} \int_{x^{*}-\epsilon}^{x^{*}+\epsilon} f_{C(t)}(x) d x \\
& =\frac{\partial}{\partial t}\left(2 \epsilon f_{C(t)}\left(x^{*}-\epsilon+\delta\right)\right) \\
& =\frac{2 \epsilon}{\pi} \frac{\partial}{\partial t}\left(\frac{t}{t^{2}+\left(x^{*}-\epsilon+\delta\right)^{2}}\right) \\
& =\frac{2 \epsilon}{\pi}\left(\frac{1}{t^{2}+\left(x^{*}-\epsilon+\delta\right)^{2}}\right. \\
& =\frac{2 \epsilon}{\pi} \frac{\left(x^{*}-\epsilon+\delta\right)^{2}-t^{2}}{\left(t^{2}+\left(x^{*}-\epsilon+\delta\right)^{2}\right)^{2}}
\end{aligned}
$$

where $\delta(0<\delta<2 \epsilon)$ may not be the same as that in (6) and (7). It is obvious that

$$
\begin{array}{ll}
\frac{\partial}{\partial t} P_{C(t)}\left(\left|x-x^{*}\right| \leq \epsilon\right)>0 & \text { if } t<\left|x^{*}-\epsilon+\delta\right| \\
\frac{\partial}{\partial t} P_{C(t)}\left(\left|x-x^{*}\right| \leq \epsilon\right)<0 & \text { if } t>\left|x^{*}-\epsilon+\delta\right| .
\end{array}
$$

That is, the larger $t$ is, the larger $P_{C(t)}\left(\left|x-x^{*}\right| \leq \epsilon\right)$ will be, if $t<\left|x^{*}-\epsilon+\delta\right|$. However, if $t>\left|x^{*}-\epsilon+\delta\right|$, the larger $t$ is, the smaller $P_{C(t)}\left(\left|x-x^{*}\right| \leq \epsilon\right)$ will be.

Since $\sigma$ and $t$ could be regarded as search step sizes for Gaussian and Cauchy mutations, the above analyses show that a large step size is beneficial (i.e., increases the probability of finding a near-optimal solution) only when the distance between the neighborhood of $x^{*}$ and the current search point (at zero) is larger than the step size or else a large step size may be detrimental to finding a near-optimal solution. The above analyses also show the rates of probability increase/decrease by deriving the explicit expressions for $\frac{\partial}{\partial \sigma} P_{G\left(0, \sigma^{2}\right)}\left(\left|x-x^{*}\right| \leq\right.$ $\epsilon)$ and $\frac{\partial}{\partial t} P_{C(t)}\left(\left|x-x^{*}\right| \leq \epsilon\right)$.

The analytical results explain why FEP achieved better results than CEP for most of the benchmark problems we tested, because the initial population was generated uniformly at random in a relatively large space and was far away from the global optimum on average. Cauchy mutation is more likely to generate larger jumps than Gaussian mutation and thus better in such cases. FEP would be less effective than CEP, however, near the small neighborhood of the global optimum because Gaussian mutation's step size is smaller (smaller is better in this case). The experimental results on functions $f_{1}$ and $f_{2}$ illustrate such behavior clearly.

The analytical results also explain why FEP with a smaller $t$ value for its Cauchy mutation would perform better whenever CEP outperforms FEP with $t=1$. If CEP outperforms FEP with $t=1$ for a problem, it implies that this FEP's search step size may be too large. In this case, using a Cauchy mutation with a smaller $t$ is very likely to improve FEP's performance since it will have a smaller search step size. The experimental results presented in Table VI match our theoretical prediction quite well.

\section{B. Empirical Evidence}

To validate the above analysis empirically, additional experiments were carried out. Function $f_{21}$ (i.e., Shekel-5) was used here since it appears to pose some difficulties to FEP. First we made the search points closer to the global optimum by generating the initial population uniformly at random in the range of $2.5 \leq x_{i} \leq 5.5$ rather than $0 \leq x_{i} \leq 10$ and repeated our previous experiments. (The global optimum of $f_{21}$ is at $x_{i}^{*}=4$.) Such minor variation to the experiment is expected to improve the performance of both CEP and FEP since the initial search points are closer to the global optimum. Note that both Gaussian and Cauchy distributions have higher probabilities in generating points around zero than those in generating points far away from zero.

The final experimental results averaged over 50 runs are given in Table VII. Fig. 6 shows the results of CEP and FEP. It is quite clear that the performance of CEP improved much more than that of FEP since the smaller average distance between search points and the global optimum favors a small step size. The mean best of CEP improved significantly from -6.86 to -7.90 , while that of FEP improved only from -5.52 to -5.62 .

Then three more sets of experiments were conducted where the search space was expanded ten times, 100 times, and 1000 times, i.e., the initial population was generated uniformly at random in the range of $0 \leq x_{i} \leq 100,0 \leq x_{i} \leq 1000$, and $0 \leq x_{i} \leq 10000$, and $a_{i}$ 's were multiplied by 10 , 100 and 1000 , respectively, making the average distance to the global optimum increasingly large. The enlarged search 
TABLE VII

Comparison of CEP's and FEP's Final Results on $f_{21}$ When the Initial Population Is Generated Uniformly at Random in the Range of $0 \leq x_{i} \leq 10$ and $2.5 \leq x_{i} \leq 5.5$. The Results Were Averaged over 50 Runs, Where "Mean Best" Indicates the Mean Best Function Values Found in the Last Generation, and "Std Dev" Stands for the Standard Deviation. The Number of Generations for Each Run Was 100

\begin{tabular}{c|cc|cc|c}
\hline Initial Range & \multicolumn{2}{|c|}{ EEP $^{\prime}$} & \multicolumn{2}{c|}{ CEP } & FEP-CEP \\
& Mean Best & Std Dev & Mean Best & Std Dev & $t$-test \\
\hline $2.5 \leq x_{i} \leq 5.5$ & -5.62 & 1.71 & -7.90 & 2.85 & $4.58^{\dagger}$ \\
\hline $0 \leq x_{i} \leq 10$ & -5.57 & 1.54 & -6.86 & 2.94 & $2.94^{\dagger}$ \\
\hline$t$-test & \multicolumn{2}{|c|}{-0.16} & \multicolumn{2}{|c|}{$-1.80^{\dagger}$} & \\
\hline
\end{tabular}

The value of $t$ with 49 degrees of freedom is significant at $\alpha=0.05$ by a two-tailed test. $\ddagger \mathrm{FEP}(\mathrm{CEP})_{\text {small }}-\mathrm{FEP}(\mathrm{CEP})_{\text {normal }}$.

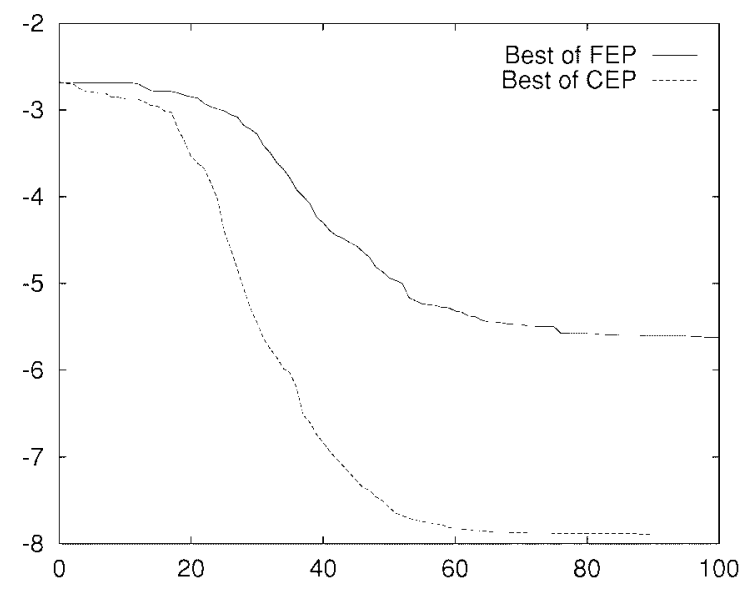

(a)

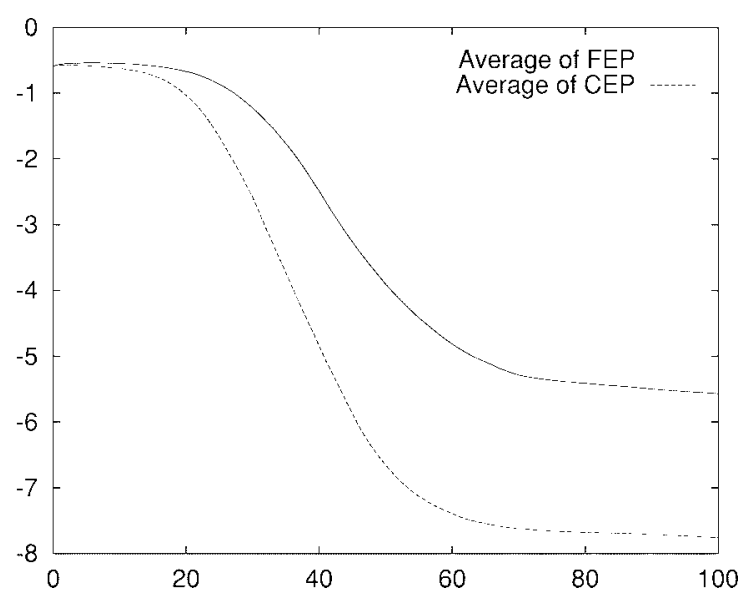

(b)

Fig. 6. Comparison between CEP and FEP on $f_{21}$ when the initial population is generated uniformly at random in the range of $2.5 \leq x_{i} \leq 5.5$. The solid lines indicate the results of FEP. The dotted lines indicate the results of CEP. (a) shows the best result, and (b) shows the average result. Both were averaged over 50 runs. The horizontal axis indicates the number of generations. The vertical axis indicates the function value.

space is expected to make the problem more difficult and thus make CEP and FEP less efficient. The results of the same experiment averaged over 50 runs are shown in Table VIII and Figs. 7-9. It is interesting to note that the performance of FEP was less affected by the larger search space than CEP. When the search space was increased to $0 \leq x_{i} \leq 100$ and $0 \leq x_{i} \leq 1000$, the superiority of CEP over FEP on $f_{21}$ disappeared. There was no statistically significant difference between CEP and FEP. When the search space was increased further to $0 \leq x_{i} \leq 10000$, FEP even outperformed CEP significantly. It is worth pointing out that a population size of 100 and the maximum number of generations of 100 are very small numbers for such a huge search space. The population might not have converged by the end of generation 100. This, however, does not affect our conclusion. The experiments still show that Cauchy mutation performs much better than Gaussian mutation when the current search points are far away from the global optimum.

Even if $a_{i}$ 's were not multiplied by 10,100 , and 1000 , similar results can still be obtained as long as the initial population was generated uniformly at random in the range of $0 \leq x_{i} \leq 100,0 \leq x_{i} \leq 1000$, and $0 \leq x_{i} \leq 10000$. Table IX shows the results when $a_{i}$ 's were unchanged. The figures of this set of experiments are omitted to save some space. It is quite clear that a similar trend can be observed as the initial ranges increase.

It is worth reiterating that the only difference between the experiments in this section and the previous experiment in Section V-C2 is the range used to generate initial random populations. The empirical results match quite well with our analysis on the relationship between the step size and the distance to the global optimum. The results also indicate that FEP is less sensitive to initial conditions than CEP and thus more robust. In practice, the global optimum is usually unknown. There is little knowledge one can use to constrain the search space to a sufficiently small region. In such cases, FEP would be a better choice than CEP.

\section{The Importance of Neighborhood Size}

It is well known that finding an exact global optimum for a multimodal function is hard without prior knowledge about the function. It might take an infinite amount of time to find the global optimum for a global search algorithm such as CEP or FEP. In practice, one often has to sacrifice discovering the global optimum in exchange for efficiency. A key issue that arises here is how much sacrifice one has to make to get a near-optimum in a reasonable amount of time. In other words, what is the relationship between the optimality of the solution 
TABLE VIII

Comparison of CEP's and FEP's Final Results on $f_{21}$ When the Initial Population Is Generated Uniformly at Random IN the RANGE OF $0 \leq x_{i} \leq 10,0 \leq x_{i} \leq 100,0 \leq x_{i} \leq 1000$, AND $0 \leq x_{i} \leq 10000$, AND $a_{i}$ 's Were Multiplied By 10, 100, AND 1000. The Results Were Averaged over 50 Runs, Where "Mean Best" Indicates the Mean Best Function Values Found in the Last Generation, and “Std Dev” Stands for the Standard Deviation. The Number of Generations for Each Run Was 100.

\begin{tabular}{|c|c|c|c|c|c|}
\hline \multirow[t]{2}{*}{ Initial Range } & \multicolumn{2}{|c|}{ FEP } & \multicolumn{2}{|c|}{ CEP } & $\mathrm{FEP}-\mathrm{CEP}$ \\
\hline & Mean Best & Std Dev & Mean Best & Std Dev & $t$-test \\
\hline $0 \leq x_{i} \leq 10000$ & -3.97 & 2.28 & -2.60 & 2.43 & $-4.02^{\dagger}$ \\
\hline $0 \leq x_{i} \leq 1000$ & -5.00 & 2.96 & -5.33 & 2.76 & 1.05 \\
\hline $0 \leq x_{i} \leq 100$ & -5.80 & 3.21 & -5.59 & 2.97 & -0.40 \\
\hline $0 \leq x_{i} \leq 10$ & -5.57 & 1.54 & -6.86 & 2.94 & $2.94^{\dagger}$ \\
\hline $\mathrm{F}(\mathrm{C}) \mathrm{EP}_{10000}-\mathrm{F}(\mathrm{C}) \mathrm{EP}_{1000}$ & \multicolumn{2}{|c|}{$2.73^{\dagger}$} & \multicolumn{2}{|c|}{$6.57^{\dagger}$} & \\
\hline $\mathrm{F}(\mathrm{C}) \mathrm{EP}_{1000}-\mathrm{F}(\mathrm{C}) \mathrm{EP}_{100}$ & \multicolumn{2}{|c|}{1.63} & \multicolumn{2}{|c|}{0.71} & \\
\hline $\mathrm{F}(\mathrm{C}) \mathrm{SP}_{100}-\mathrm{F}(\mathrm{C}) \mathrm{EP}_{10}$ & \multicolumn{2}{|c|}{-0.48} & \multicolumn{2}{|c|}{$2.10^{\dagger}$} & \\
\hline
\end{tabular}

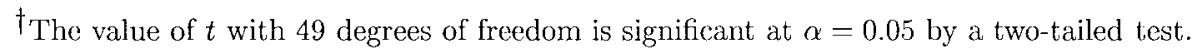

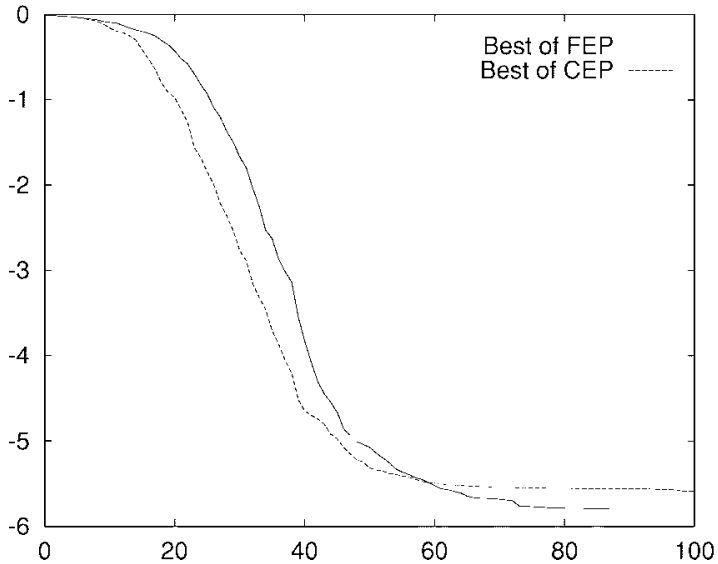

(a)

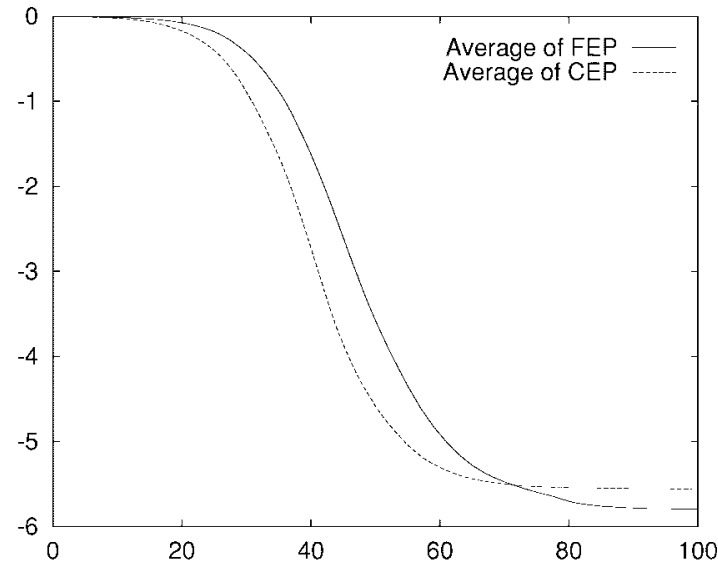

(b)

Fig. 7. Comparison between CEP and FEP on $f_{21}$ when the initial population is generated uniformly at random in the range of $0 \leq x_{i} \leq 100$ and $a_{i}$ 's were multiplied by ten. The solid lines indicate the results of FEP. The dotted lines indicate the results of CEP. (a) shows the best result, and (b) shows the average result. Both were averaged over 50 runs. The horizontal axis indicates the number of generations. The vertical axis indicates the function value.

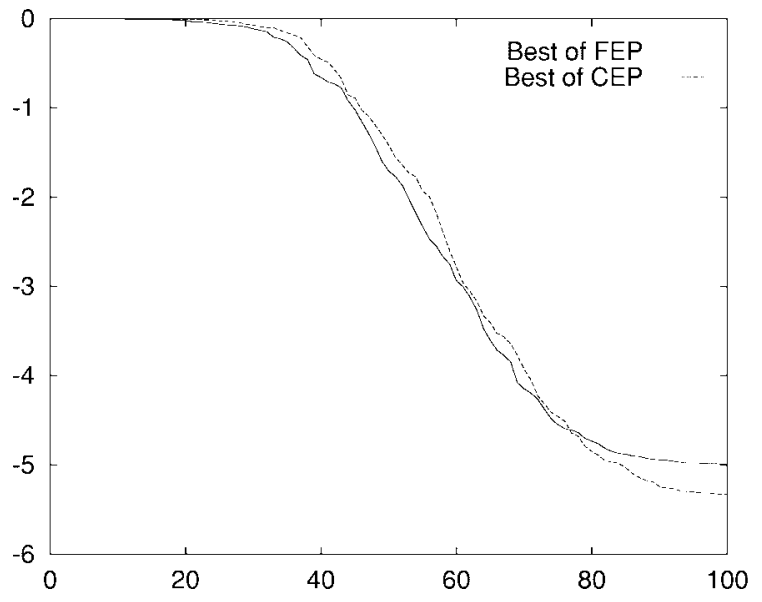

(a)

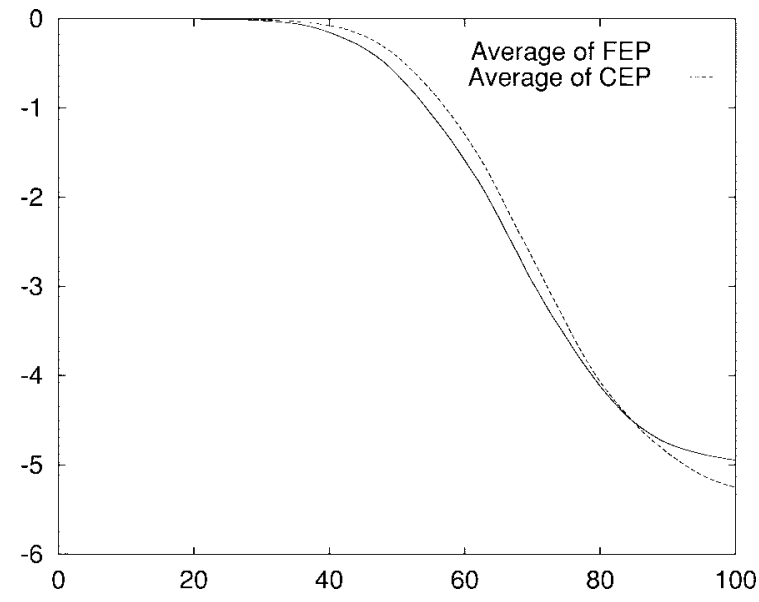

(b)

Fig. 8. Comparison between CEP and FEP on $f_{21}$ when the initial population is generated uniformly at random in the range of $0 \leq x_{i} \leq 1000$ and $a_{i}$ 's were multiplied by 100. The solid lines indicate the results of FEP. The dotted lines indicate the results of CEP. (a) shows the best result, and (b) shows the average result. Both were averaged over 50 runs. The horizontal axis indicates the number of generations. The vertical axis indicates the function value. 

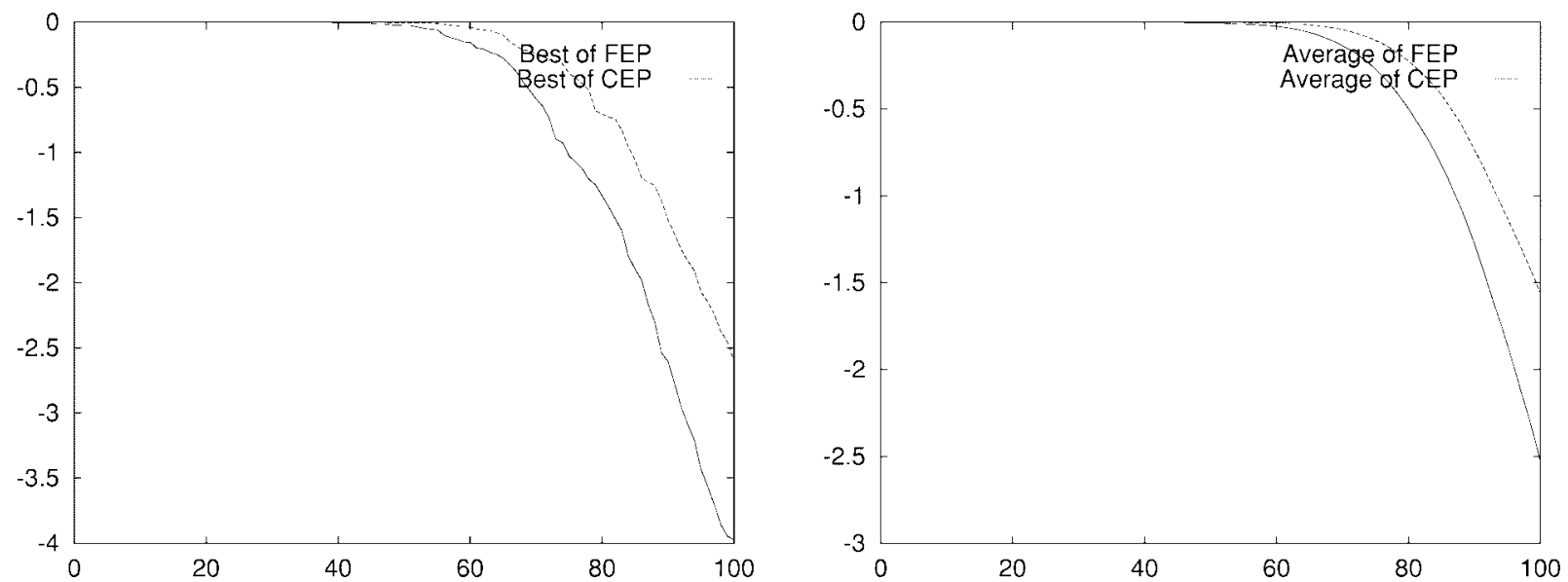

Fig. 9. Comparison between CEP and FEP on $f_{21}$ when the initial population is generated uniformly at random in the range of $0 \leq x_{i} \leq 10000$ and $a_{i}$ 's were multiplied by 1000. The solid lines indicate the results of FEP. The dotted lines indicate the results of CEP. (a) shows the best result, and (b) shows the average result. Both were averaged over 50 runs. The horizontal axis indicates the number of generations. The vertical axis indicates the function value.

TABLE IX

Comparison of CEP's and FEP's Final Results on $f_{21}$ When the Initial Population Is Generated Uniformly at Random in THE RANGe OF $0 \leq x_{i} \leq 10,0 \leq x_{i} \leq 100,0 \leq x_{i} \leq 1000$, and $0 \leq x_{i} \leq 10000 . a_{i}$ 's Were UnChANGed. THE

Results Were Averaged over 50 Runs, Where "Mean Best" Indicates the Mean Best Function Values Found in the Last Generation, and "Std Dev" Stands for the Standard Deviation. The Number of Generations for Each Run Was 100

\begin{tabular}{c|cc|cc|c}
\hline Initial Range & \multicolumn{2}{|c|}{ FEP } & \multicolumn{2}{c|}{ CEP } & FEP-CEP \\
& Mean Best & Std Dev & Mean Best & Std Dev & $t$-test \\
\hline $0 \leq x_{i} \leq 10000$ & -4.12 & 1.45 & -1.40 & 1.43 & $-10.44^{\dagger}$ \\
\hline $0 \leq x_{i} \leq 1000$ & -5.10 & 0.81 & -5.15 & 1.66 & 0.19 \\
\hline $0 \leq x_{i} \leq 100$ & -5.16 & 1.13 & -5.61 & 2.31 & 1.21 \\
\hline $0 \leq x_{i} \leq 10$ & -5.57 & 1.54 & -6.86 & 2.94 & $2.94^{\dagger}$ \\
\hline $\mathrm{F}(\mathrm{C}) \mathrm{EP}_{10000}-\mathrm{F}(\mathrm{C}) \mathrm{EP}_{1000}$ & \multicolumn{2}{|c|}{$4.72^{\dagger}$} & \multicolumn{2}{|c|}{$12.46^{\dagger}$} & \\
\hline $\mathrm{F}(\mathrm{C}) \mathrm{EP} 1000-\mathrm{F}(\mathrm{C}) \mathrm{EP}_{100}$ & \multicolumn{2}{|c|}{0.33} & \multicolumn{2}{|c|}{1.33} & \\
\hline $\mathrm{F}(\mathrm{C}) \mathrm{EP}_{100}-\mathrm{F}(\mathrm{C}) \mathrm{EP}_{10}$ & \multicolumn{2}{|c|}{1.48} & $2.24^{\dagger}$ & \\
\hline
\end{tabular}

tThe value of $t$ with 49 degrees of freedom is significant at $\alpha=0.05$ by a two-tailed test.

and the time used to find the solution? This issue can be approached from the point of view of neighborhood size, i.e., $\epsilon$ in (5), since a smaller neighborhood size usually implies better optimality. It will be very useful if the impact of neighborhood size $\epsilon$ on the probability of generating a near-optimum in that neighborhood can be worked out. (The probability of finding a near-optimum would be the same as that of generating it when the elitism is used.) Although not an exact answer to the issue, the following analysis does provide some insights into such impact.

Similar to the analysis in Section VII-A7, the following is true according to the mean value theorem for definite integrals [17, p. 322]: for $0<\delta<2 \epsilon \mathrm{f}$

$$
\begin{aligned}
\frac{\partial}{\partial \epsilon} & P_{G\left(0, \sigma^{2}\right)}\left(\left|x-x^{*}\right| \leq \epsilon\right) \\
& =\frac{\partial}{\partial \epsilon} \int_{x^{*}-\epsilon}^{x^{*}+\epsilon} f_{G\left(0, \sigma^{2}\right)}(x) d x \\
& =\frac{\partial}{\partial \epsilon}\left(2 \epsilon f_{G\left(0, \sigma^{2}\right)}\left(x^{*}-\epsilon+\delta\right)\right) \\
& =\frac{\partial}{\partial \epsilon}\left(2 \epsilon \frac{1}{\sigma \sqrt{2 \pi}} e^{-\frac{\left(x^{*}-c+\delta\right)^{2}}{2 \sigma^{2}}}\right)
\end{aligned}
$$

$$
\begin{aligned}
& =\frac{2}{\sigma \sqrt{2 \pi}} \frac{\partial}{\partial \epsilon}\left(\epsilon e^{\left.-\frac{\left(x^{*}-c+\delta\right)^{2}}{2 \sigma^{2}}\right)}\right. \\
& =\frac{2}{\sigma \sqrt{2 \pi}} e^{-\frac{\left(x^{*}-c+\delta\right)^{2}}{2 \sigma^{2}}}\left(1+\frac{\epsilon}{\sigma^{2}}\left(x^{*}-\epsilon+\delta\right)\left(1-\frac{\partial \delta}{\partial \epsilon}\right)\right) .
\end{aligned}
$$

For the above equation, there exists a sufficiently small number $\epsilon_{1}>0$ such that for any $\epsilon \leq \epsilon_{1}$

$$
\left|\frac{\epsilon}{\sigma^{2}}\left(x^{*}-\epsilon+\delta\right)\left(1-\frac{\partial \delta}{\partial \epsilon}\right)\right|<1 .
$$

That is, for $0<\epsilon \leq \epsilon_{1}$

$$
\frac{\partial}{\partial \epsilon} P_{G\left(0, \sigma^{2}\right)}\left(\left|x-x^{*}\right| \leq \epsilon\right)>0
$$

which implies that the probability of generating a nearoptimum increases as the neighborhood size increases in the vicinity of the optimum. The rate of such probability growth (i.e., $\frac{\partial}{\partial \epsilon} P_{G\left(0, \sigma^{2}\right)}\left(\left|x-x^{*}\right| \leq \epsilon\right)$ ) is governed by the term

$$
e^{-\frac{\left(x^{*}-c+\delta\right)^{2}}{2 \sigma^{2}}}=e^{-\frac{\left(x^{*}-(c-\delta)\right)^{2}}{2 \sigma^{2}}}
$$

That is, $\frac{\partial}{\partial \epsilon} P_{G\left(0, \sigma^{2}\right)}\left(\left|x-x^{*}\right| \leq \epsilon\right)$ grows exponentially faster as $\epsilon-\delta$ increases. 
TABLE X

Comparison Among IFEP, FEP, and CEP on Functions $f_{1}, f_{2}, f_{10}, f_{11}, f_{21}, f_{22}$, and $f_{23}$. All Results Have BeEn Averaged over 50 Runs, Where "Mean Best" Indicates the Mean Best Function Values Found in the Last Generation

\begin{tabular}{llccccc}
\hline F & $\begin{array}{c}\text { \# of } \\
\text { Gen's }\end{array}$ & $\begin{array}{c}\text { IFEP } \\
\text { Mean Best }\end{array}$ & $\begin{array}{c}\text { FEP } \\
\text { Mean Best }\end{array}$ & $\begin{array}{c}\text { CEP } \\
\text { Mean Best }\end{array}$ & $\begin{array}{c}\text { IFEP-FEP } \\
t \text {-tost }\end{array}$ & $\begin{array}{c}\text { IFEP-CEP } \\
t \text {-test }\end{array}$ \\
\hline$f_{1}$ & 1500 & $4.16 \times 10^{-5}$ & $5.72 \times 10^{-4}$ & $1.91 \times 10^{-4}$ & $-28.06^{\dagger}$ & $-2.39^{\dagger}$ \\
$f_{2}$ & 2000 & $2.44 \times 10^{-2}$ & $7.60 \times 10^{-2}$ & $2.29 \times 10^{-2}$ & $-51.61^{\dagger}$ & $3.47^{\dagger}$ \\
$f_{10}$ & 1500 & $4.83 \times 10^{-3}$ & $1.76 \times 10^{-2}$ & 8.79 & $-48.54^{\dagger}$ & $-21.26^{\dagger}$ \\
$f_{11}$ & 2000 & $4.54 \times 10^{-2}$ & $2.49 \times 10^{-2}$ & $8.13 \times 10^{-2}$ & $2.16^{\dagger}$ & $-2.19^{\dagger}$ \\
$f_{21}$ & 100 & -6.46 & -5.50 & -6.43 & $-2.19^{\dagger}$ & $-5.46^{\dagger}$ \\
$f_{22}$ & 100 & -7.10 & -5.73 & -7.62 & $-2.25^{\dagger}$ & 0.84 \\
$f_{23}$ & 100 & -7.80 & -6.41 & -8.86 & $-2.14^{\dagger}$ & $1.73^{\dagger}$ \\
\hline
\end{tabular}

${ }^{\dagger}$ The value of $t$ with 49 degrees of freedom is significant at $\alpha=0.05$ by a two-tailed test.

A similar analysis can be carried out for Cauchy mutation using its density function, i.e., (3). Let the density function be $C(1)$ when $t=1$. For $0<\delta<2 \epsilon$

$$
\begin{aligned}
\frac{\partial}{\partial \epsilon} & P_{C(1)}\left(\left|x-x^{*}\right| \leq \epsilon\right) \\
& =\frac{\partial}{\partial \epsilon} \int_{x^{*}-\epsilon}^{x^{*}+\epsilon} f_{C(1)}(x) d x \\
& =\frac{\partial}{\partial \epsilon} \int_{x^{*}-\epsilon}^{x^{*}+\epsilon} \frac{1}{\pi\left(1+x^{2}\right)} d x \\
& =\frac{\partial}{\partial \epsilon}\left(\frac{1}{\pi}\left(\arctan \left(x^{*}+\epsilon\right)-\arctan \left(x^{*}-\epsilon\right)\right)\right) \\
& =\frac{1}{\pi}\left(\frac{1}{1+\left(x^{*}+\epsilon\right)^{2}}+\frac{1}{1+\left(x^{*}-\epsilon\right)^{2}}\right) \\
& >0 .
\end{aligned}
$$

Hence the probability of generating a near-optimum in the neighborhood always increases as the neighborhood size increases. While this conclusion is quite straightforward, it is interesting to note that the rate of increase in the probability differs significantly between Gaussian and Cauchy mutation since $\frac{\partial}{\partial \epsilon} P_{C(1)}\left(\left|x-x^{*}\right| \leq \epsilon\right) \gg \frac{\partial}{\partial \epsilon} P_{G(0,1)}\left(\left|x-x^{*}\right| \leq \epsilon\right)$.

\section{AN IMPRoved FAST EVOlutionary Programming}

The previous analyses show the benefits of FEP and CEP in different situations. Generally, Cauchy mutation performs better when the current search point is far away from the global minimum, while Gaussian mutation is better at finding a local optimum in a good region. It would be ideal if Cauchy mutation is used when search points are far away from the global optimum and Gaussian mutation is adopted when search points are in the neighborhood of the global optimum. Unfortunately, the global optimum is usually unknown in practice, making the ideal switch from Cauchy to Gaussian mutation very difficult. Self-adaptive Gaussian mutation [7], [2], [8] is an excellent technique to partially address the problem. That is, the evolutionary algorithm itself will learn when to "switch" from one step size to another. There is room for further improvement, however, to self-adaptive algorithms like CEP or even FEP.
This paper proposes an improved FEP (IFEP) based on mixing (rather than switching) different mutation operators. The idea is to mix different search biases of Cauchy and Gaussian mutations. The importance of search biases has been pointed out by some earlier studies [18, pp. 375-376]. The implementation of IFEP is very simple. It differs from FEP and CEP only in Step 3 of the algorithm described in Section II. Instead of using (1) (for CEP) or (4) (for FEP) alone, IFEP generates two offspring from each parent, one by Cauchy mutation and the other by Gaussian. The better one is then chosen as the offspring. The rest of the algorithm is exactly the same as FEP and CEP. Chellapilla [19] has recently presented some more results on comparing different mutation operators in EP.

\section{A. Experimental Studies}

To carry out a fair comparison among IFEP, FEP, and CEP, the population size of IFEP was reduced to half of that of FEP or CEP in all the following experiments, since each individual in IFEP generates two offspring. Reducing IFEP's population size by half, however, actually puts IFEP at a slight disadvantage because it does not double the time for any operators (such as selection) other than mutations. Nevertheless, such comparison offers a good and simple compromise.

IFEP was tested in the same experimental setup as before. For the sake of clarity and brevity, only some representative functions (out of 23) from each group were tested. Functions $f_{1}$ and $f_{2}$ are typical unimodal functions. Functions $f_{10}$ and $f_{11}$ are multimodal functions with many local minima. Functions $f_{21}-f_{23}$ are multimodal functions with only a few local minima and are particularly challenging to FEP. Table X summarizes the final results of IFEP in comparison with FEP and CEP. Figs. 10 and 11 show the results of IFEP, FEP, and CEP.

\section{B. Discussions}

It is very clear from Table $\mathrm{X}$ that IFEP has improved FEP's performance significantly for all test functions except for $f_{11}$. Even in the case of $f_{11}$, IFEP is better than FEP for 25 out of 50 runs. In other words, IFEP's performance is still rather close to FEP's and certainly better than CEP's (35 out of 50 runs) on $f_{11}$. These results show that IFEP continues to perform 

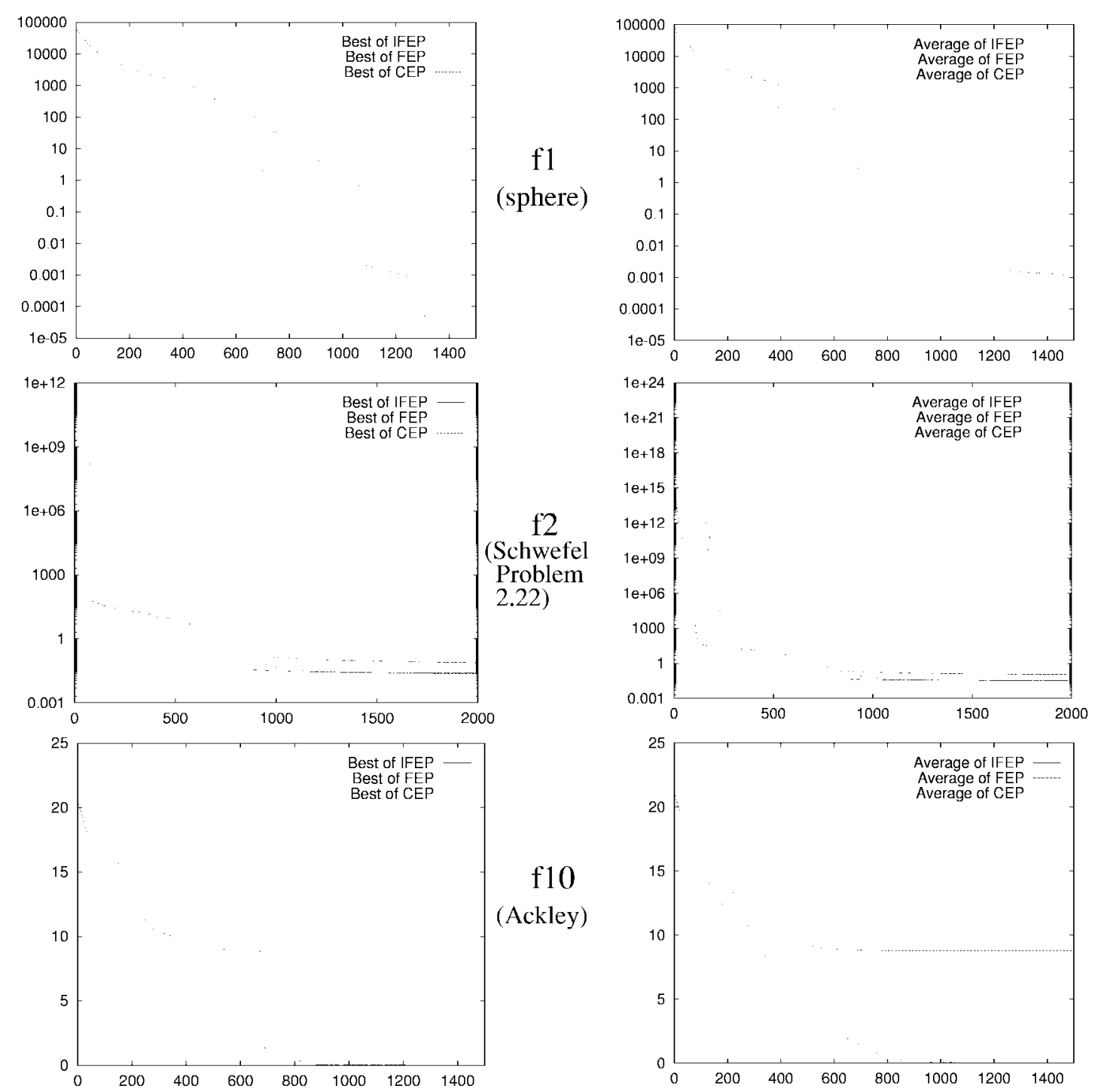

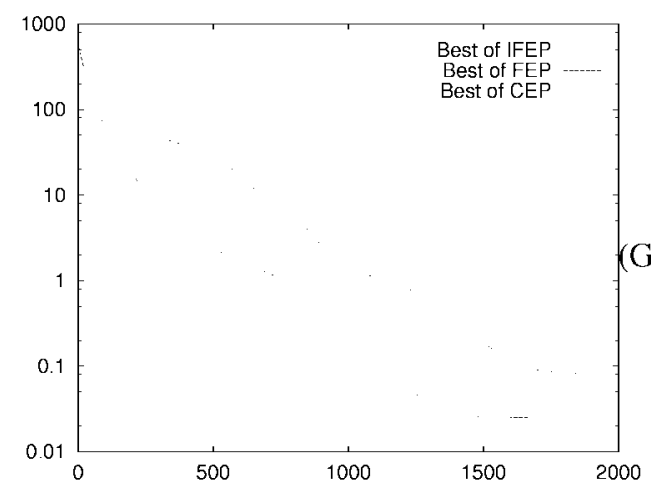

(a)

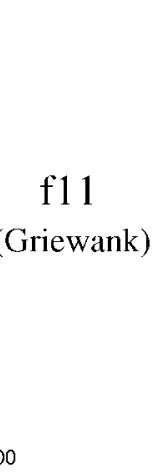

Fig. 10. Comparison among IFEP, FEP, and CEP on functions $f_{1}, f_{2}, f_{10}$, and $f_{11}$. The vertical axis is the function value, and the horizontal axis is the number of generations. The solid lines indicate the results of IFEP. The dashed lines indicate the results of FEP. The dotted lines indicate the results of CEP. (a) shows the best results, and (b) shows the average results. All were averaged over 50 runs.

at least as well as FEP on multimodal functions with many minima and also performs very well on unimodal functions and multimodal functions with only a few local minima with which FEP has difficulty handling. IFEP achieved performance similar to CEP's on these functions.

For the two unimodal functions where FEP is outperformed by CEP significantly, IFEP performs better than CEP on $f_{1}$, while worse than CEP on $f_{2}$. A closer look at the actual average solutions reveals that IFEP found much better solution than CEP on $f_{1}$ (roughly an order of magnitude smaller) while only performed slightly worse than CEP on $f_{2}$.

For the three Shekel functions $f_{21}-f_{23}$, the difference between IFEP and CEP is much smaller than that between FEP and CEP. IFEP has improved FEP's performance significantly 

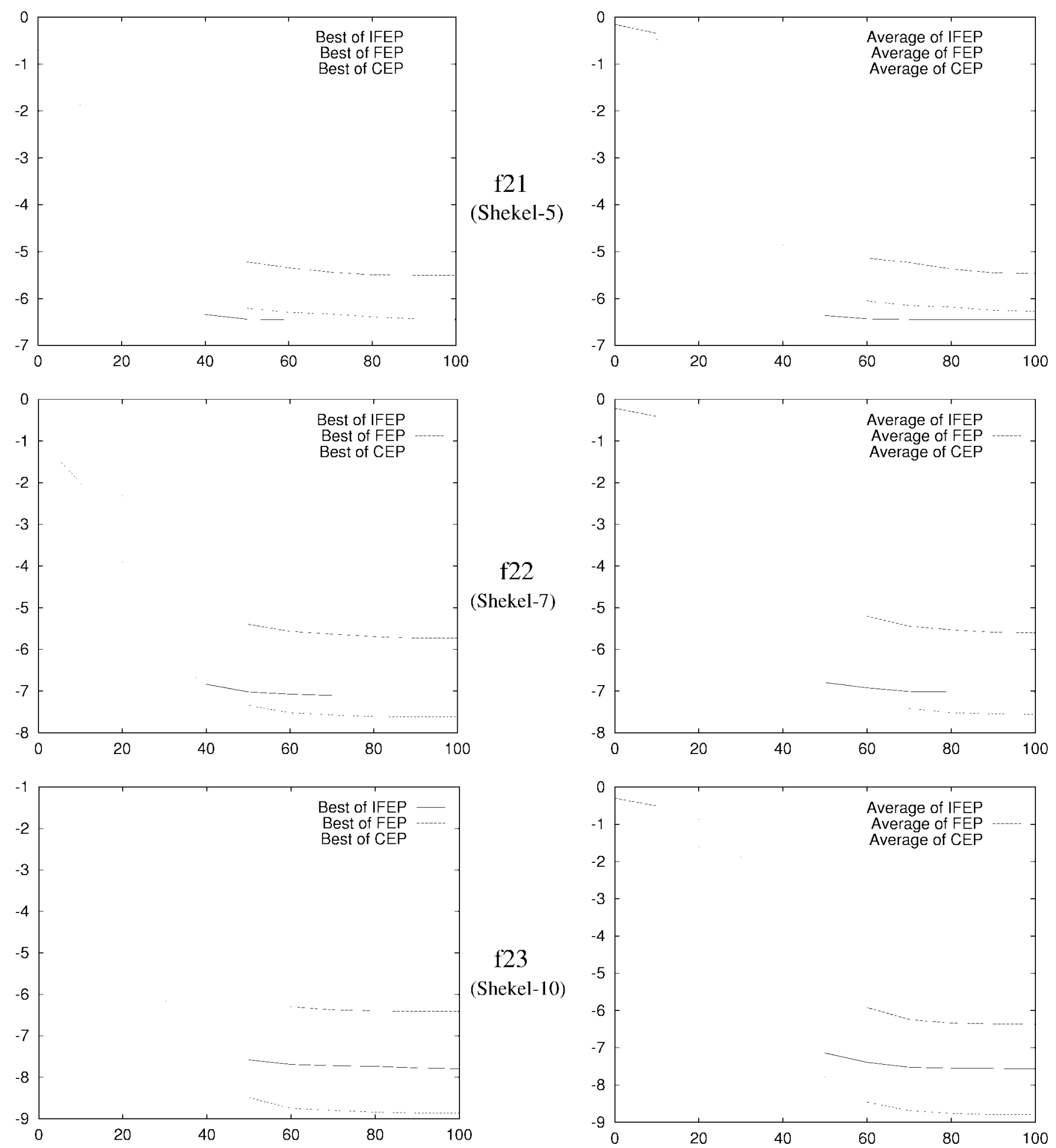

f23

(Shekel-10)

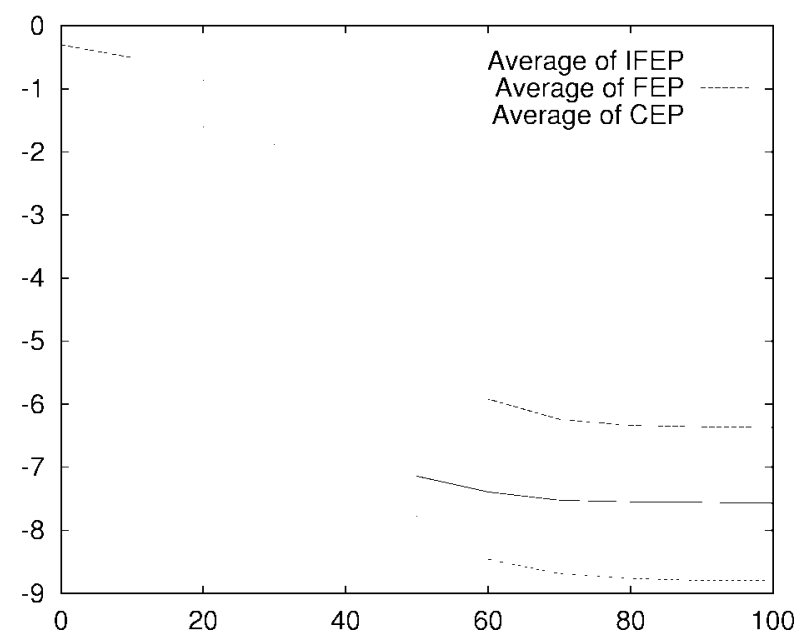

Fig. 11. Comparison among IFEP, FEP and CEP on functions $f_{21}-f_{23}$. The vertical axis is the function value, and the horizontal axis is the number of generations. The solid lines indicate the results of IFEP. The dashed lines indicate the results of FEP. The dotted lines indicate the results of CEP. (a) shows the best results, and (b) shows the average results. All were averaged over 50 runs.

on all three functions. It performs better than $\mathrm{CEP}$ on $f_{21}$, the same on $f_{22}$, and worse on $f_{23}$.

It is very encouraging that IFEP is capable of performing as well as or better than the better one of FEP and CEP for most test functions. This is achieved through a minimal change to the existing FEP and CEP. No prior knowledge or any other complicated operators were used. There is no additional parameter used either. The superiority of IFEP also demonstrates the importance of mixing difference search biases (e.g., "step sizes") in a robust search algorithm.

The population size of IFEP used in the above experiments was only half of that of FEP and CEP. It is not unreasonable to expect even better results from IFEP if it uses the same population size as FEP's and CEP's. For $(\mu, \lambda)$ or $(\mu+\lambda)$ 


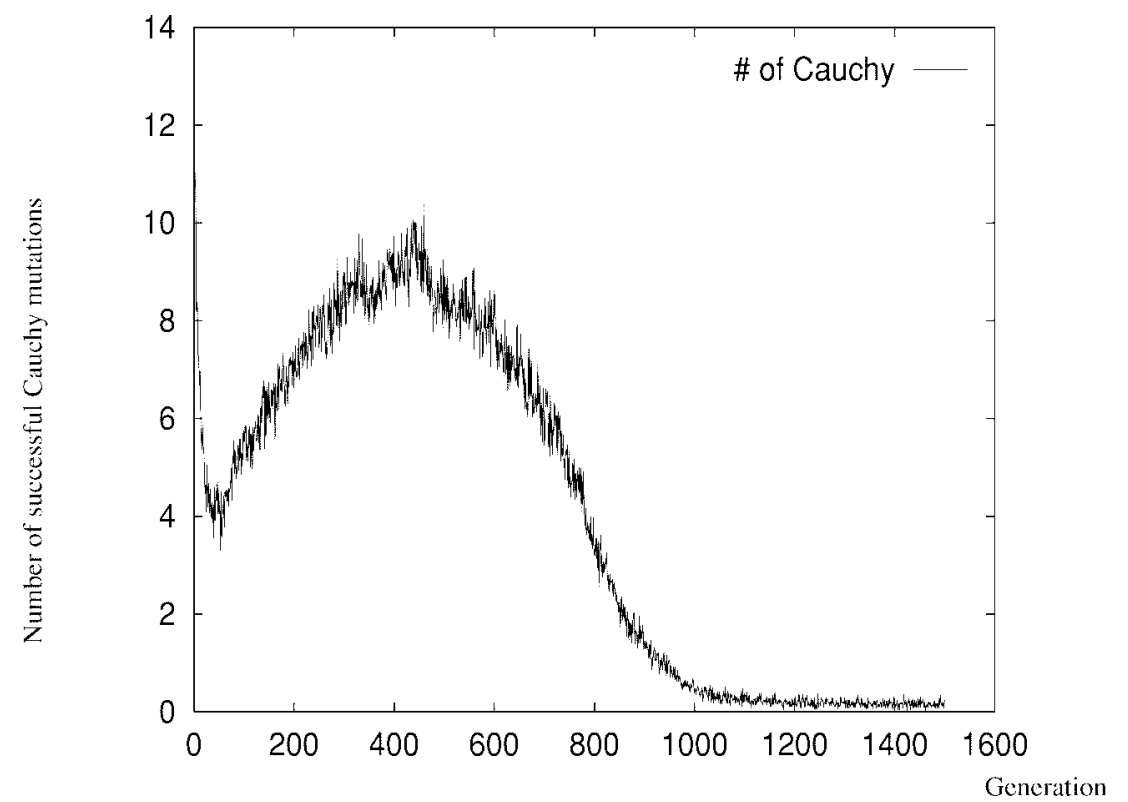

Fig. 12. Number of successful Cauchy mutations in a population when IFEP is applied to function $f_{1}$. The vertical axis indicates the number of successful Cauchy mutations in a population, and the horizontal axis indicates the number of generations. The results have been averaged over 50 runs.

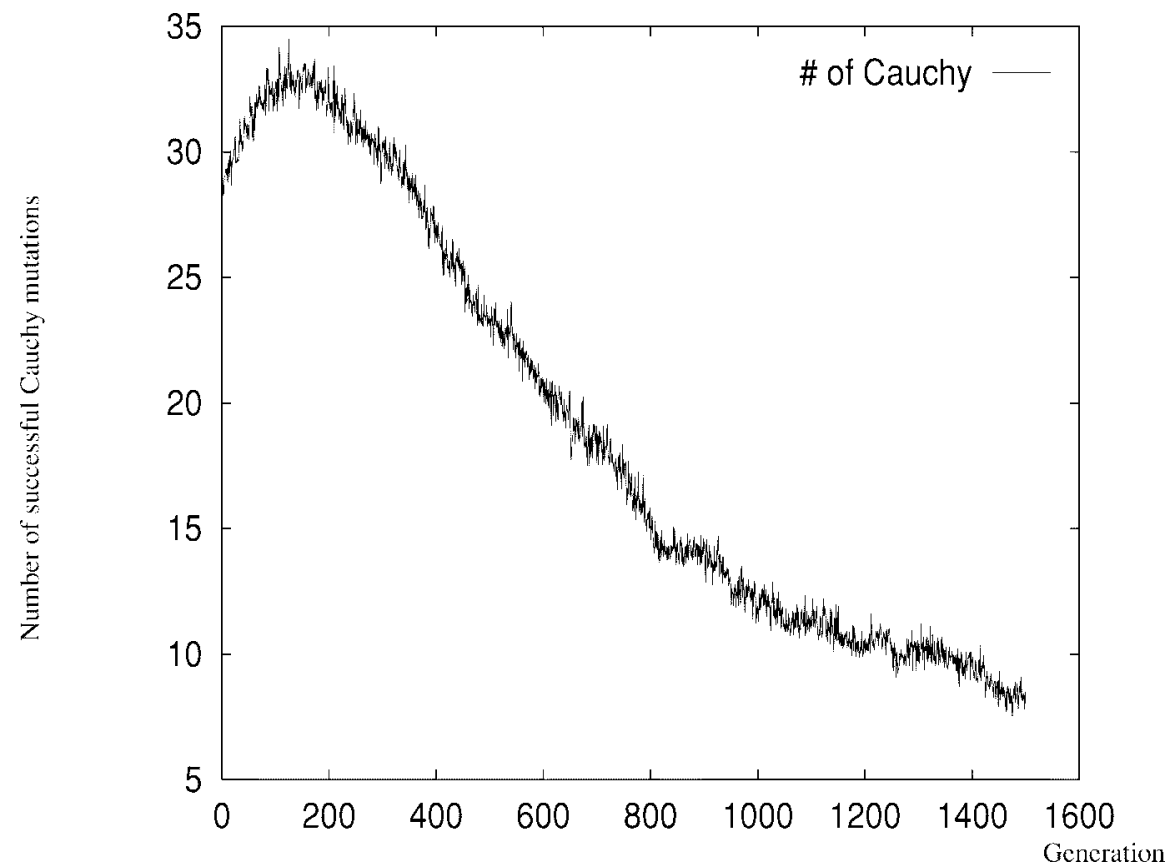

Fig. 13. Number of successful Cauchy mutations in a population when IFEP is applied to function $f_{10}$. The vertical axis indicates the number of successful Cauchy mutations in a population, and the horizontal axis indicates the number of generations. The results have been averaged over 50 runs.

evolutionary algorithms where $\mu<\lambda$, it would be quite natural to use both Cauchy and Gaussian mutations since a parent needs to generate more than one offspring anyway.

It has been mentioned several times in this paper that Cauchy mutation performs better than Gaussian mutation because of its higher probability of making large jumps (i.e., having a larger expected search step size). According to our theoretical analysis, however, large search step sizes would be detrimental to search when the current search points are very close to the global optimum. Figs. 12-14 show the number of successful Cauchy mutations in a population in different generations. It is obvious that Cauchy mutation played a major role in the population in the early stages of evolution since the distance between the current search points and the global optimum was relatively large on average in the early stages. Hence Cauchy mutation performed better. As the evolution progressed, however, the distance became smaller and smaller. Large search step sizes produced by Cauchy mutation tended to produce worse offspring than those produced by Gaussian mutation. The decreasing number of successful Cauchy mutations in those figures illustrates this behavior. 


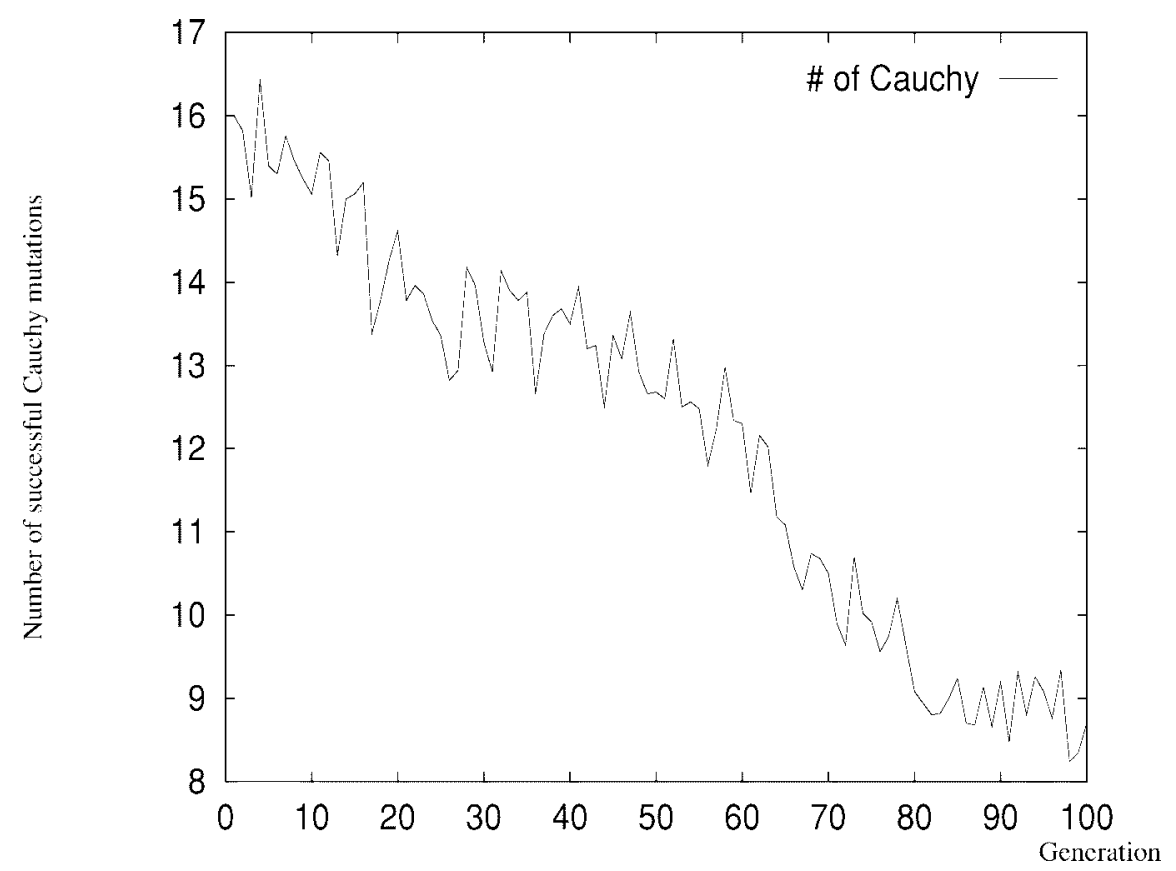

Fig. 14. Number of successful Cauchy mutations in a population when IFEP is applied to function $f_{21}$. The vertical axis indicates the number of successful Cauchy mutations in a population, and the horizontal axis indicates the number of generations. The results have been averaged over 50 runs.

\section{CONCLUSION}

This paper first proposes a fast evolutionary programming algorithm FEP and evaluates its performance on a number of benchmark problems. The experimental results show that FEP performs much better than CEP for multimodal functions with many local minima while being comparable to CEP in performance for unimodal and multimodal functions with only a few local minima. Since FEP and CEP differ only in their mutations, it is quite easy to apply FEP to real-world problems. No additional cost was introduced except for the difference in generating a Cauchy random number instead of a Gaussian random number.

The paper then analyzes FEP and CEP in depth in terms of search step size and neighborhood size and explains why FEP performs better than CEP for most benchmark problems. The theoretical analysis is supported by the additional empirical evidence in which the range of initial $x$ values was changed. The paper shows that FEP's long jumps increase the probability of finding a near-optimum when the distance between the current search point and the optimum is large, but decrease the probability when such distance is small. The paper also investigates the relationship between the neighborhood size and the probability of finding a near-optimum in this neighborhood. Some insights on evolutionary search and optimization in general have been gained from the above analyses.

The above analyses also led to an IFEP which is very simple yet effective. IFEP uses the idea of mixing search biases to mix Cauchy and Gaussian mutations. Unlike some switching algorithms which have to decide when to switch between different mutations during search, IFEP does not need to make such decision and introduces no parameters. IFEP is robust, assumes no prior knowledge of the problem to be solved, and performs at least as well as the better one of FEP and CEP for most benchmark problems. Future work on IFEP includes the comparison of IFEP with other self-adaptive algorithms such as [20] and other evolutionary algorithms using Cauchy mutation [21].

The idea of FEP and IFEP can also be applied to other evolutionary algorithms to design faster optimization algorithms [22]. For $(\mu+\lambda)$ and $(\mu, \lambda)$ evolutionary algorithms where $\mu<\lambda$, IFEP would be particularly attractive since a parent has to generate more than one offspring. It may be beneficial if different offspring are generated by different mutations [22].

\section{APPENDIX \\ BENCHMARK FUNCTIONS}

\section{A. Sphere Model}

$$
\begin{array}{r}
f_{1}(x)=\sum_{i=1}^{30} x_{i}^{2}, \quad-100 \leq x_{i} \leq 100 \\
\min \left(f_{1}\right)=f_{1}(0, \ldots, 0)=0 .
\end{array}
$$

B. Schwefel's Problem 2.22

$$
\begin{gathered}
f_{2}(x)=\sum_{i=1}^{30}\left|x_{i}\right|+\prod_{i=1}^{30}\left|x_{i}\right| \quad-10 \leq x_{i} \leq 10 \\
\min \left(f_{2}\right)=f_{2}(0, \ldots, 0)=0 .
\end{gathered}
$$

C. Schwefel's Problem 1.2

$$
\begin{gathered}
f_{3}(x)=\sum_{i=1}^{30}\left(\sum_{j=1}^{i} x_{j}\right)^{2}, \quad-100 \leq x_{i} \leq 100 \\
\min \left(f_{3}\right)=f_{3}(0, \ldots, 0)=0 .
\end{gathered}
$$


D. Schwefel's Problem 2.21

$$
\begin{gathered}
f_{4}(x)=\max _{i}\left\{\left|x_{i}\right|, 1 \leq i \leq 30\right\}, \quad-100 \leq x_{i} \leq 100 \\
\min \left(f_{4}\right)=f_{4}(0, \ldots, 0)=0 .
\end{gathered}
$$

E. Generalized Rosenbrock's Function

$$
\begin{aligned}
f_{5}(x)= & \sum_{i=1}^{29}\left[100\left(x_{i+1}-x_{i}^{2}\right)^{2}+\left(x_{i}-1\right)^{2}\right], \\
& -30 \leq x_{i} \leq 30 \quad \min \left(f_{5}\right)=f_{5}(1, \ldots, 1)=0 .
\end{aligned}
$$

F. Step Function

$$
\begin{gathered}
f_{6}(x)=\sum_{i=1}^{30}\left(\left\lfloor x_{i}+0.5\right\rfloor\right)^{2} \quad-100 \leq x_{i} \leq 100 \\
\min \left(f_{6}\right)=f_{6}(0, \ldots, 0)=0
\end{gathered}
$$

G. Quartic Function i.e. Noise

$$
\begin{gathered}
f_{7}(x)=\sum_{i=1}^{30} i x_{i}^{4}+\operatorname{random}[0,1) \quad-1.28 \leq x_{i} \leq 1.28 \\
\min \left(f_{7}\right)=f_{7}(0, \ldots, 0)=0 .
\end{gathered}
$$

H. Generalized Schwefel's Problem 2.26

$$
\begin{aligned}
f_{8}(x)= & -\sum_{i=1}^{30}\left(x_{i} \sin \left(\sqrt{\left|x_{i}\right|}\right)\right) \quad-500 \leq x_{i} \leq 500 \\
& \min \left(f_{8}\right)=f_{8}(420.9687, \ldots, 420.9687)=-12569.5 .
\end{aligned}
$$

\section{Generalized Rastrigin's Function}

$$
\begin{aligned}
f_{9}(x)= & \left.\sum_{i=1}^{30}\left[x_{i}^{2}-10 \cos \left(2 \pi x_{i}\right)+10\right)\right], \\
& -5.12 \leq x_{i} \leq 5.12 \quad \min \left(f_{9}\right)=f_{9}(0, \ldots, 0)=0 .
\end{aligned}
$$

\section{J. Ackley's Function}

$$
\begin{aligned}
f_{10}(x) & =-20 \exp \left(-0.2 \sqrt{\frac{1}{30} \sum_{i=1}^{30} x_{i}^{2}}\right) \\
& -\exp \left(\frac{1}{30} \sum_{i=1}^{30} \cos 2 \pi x_{i}\right)+20+e \\
& -32 \leq x_{i} \leq 32, \quad \min \left(f_{10}\right)=f_{10}(0, \ldots, 0)=0 .
\end{aligned}
$$

TABLE XI

KOWALIK'S FUNCTION $f_{15}$

\begin{tabular}{c|c|c}
\hline $\mathrm{i}$ & $a_{i}$ & $b_{i}^{-{ }^{-}}$ \\
\hline 1 & 0.1957 & 0.25 \\
2 & 0.1947 & 0.5 \\
3 & 0.1735 & 1 \\
4 & 0.1600 & 2 \\
5 & 0.0844 & 4 \\
6 & 0.0627 & 6 \\
7 & 0.0456 & 8 \\
8 & 0.0342 & 10 \\
9 & 0.0323 & 12 \\
10 & 0.0235 & 14 \\
11 & 0.0246 & 16 \\
\hline
\end{tabular}

TABLE XII

HARTMAN FUNCTION $f_{19}$

\begin{tabular}{c|ccc|c|ccc}
\hline $\mathrm{i}$ & \multicolumn{3}{|c|}{$a_{i j}, j=1,2,3$} & $c_{i}$ & \multicolumn{3}{|c}{$p_{i j}, j=1,2,3$} \\
\hline 1 & 3 & 10 & 30 & 1 & 0.3689 & 0.1170 & 0.2673 \\
2 & 0.1 & 10 & 35 & 1.2 & 0.4699 & 0.4387 & 0.7470 \\
3 & 3 & 10 & 30 & 3 & 0.1091 & 0.8732 & 0.5547 \\
4 & 0.1 & 10 & 35 & 3.2 & 0.038150 & 0.5743 & 0.8828 \\
\hline
\end{tabular}

\section{K. Generalized Griewank Function}

$$
\begin{array}{r}
f_{11}(x)=\frac{1}{4000} \sum_{i=1}^{30} x_{i}^{2}-\prod_{i=1}^{30} \cos \left(\frac{x_{i}}{\sqrt{i}}\right)+1 \\
-600 \leq x_{i} \leq 600 \quad \min \left(f_{11}\right)=f_{11}(0, \ldots, 0)=0 .
\end{array}
$$

L. Generalized Penalized Functions

$$
\begin{aligned}
f_{12}(x)= & \frac{\pi}{30}\left\{10 \sin ^{2}\left(\pi y_{1}\right)+\sum_{i=1}^{29}\left(y_{i}-1\right)^{2}\right. \\
& \left.\cdot\left[1+10 \sin ^{2}\left(\pi y_{i+1}\right)\right]+\left(y_{n}-1\right)^{2}\right\} \\
+ & \sum_{i=1}^{30} u\left(x_{i}, 10,100,4\right)
\end{aligned}
$$

$$
\begin{aligned}
-50 \leq x_{i} \leq 50, \quad \min \left(f_{12}\right)=f_{12}(1, \ldots, 1)=0 \\
f_{13}(x)=0.1\left\{\sin ^{2}\left(\pi 3 x_{1}\right)+\sum_{i=1}^{29}\left(x_{i}-1\right)^{2}\left[1+\sin ^{2}\right.\right. \\
\left.\left.\cdot\left(3 \pi x_{i+1}\right)\right]+\left(x_{n}-1\right)^{2}\left[1+\sin ^{2}\left(2 \pi x_{30}\right)\right]\right\}
\end{aligned}
$$$$
+\sum_{i=1}^{30} u\left(x_{i}, 5,100,4\right)
$$

$-50 \leq x_{i} \leq 50, \quad \min \left(f_{13}\right)=f_{13}(1, \ldots, 1)=0$

where

$$
\begin{aligned}
u\left(x_{i}, a, k, m\right) & = \begin{cases}k\left(x_{i}-a\right)^{m}, & x_{i}>a \\
0, & -a \leq x_{i} \leq a, \\
k\left(-x_{i}-a\right)^{m}, & x_{i}<-a\end{cases} \\
y_{i} & =1+\frac{1}{4}\left(x_{i}+1\right) .
\end{aligned}
$$


TABLE XIII

HARTMAN FUNCTION $f_{20}$

\begin{tabular}{c|cccccc|c|cccccc}
\hline $\mathrm{i}$ & \multicolumn{6}{|c|}{$a_{i j}, j=1, \cdots, 6$} & $c_{i}$ & \multicolumn{7}{c}{$p_{i j}, j=1, \cdots, 6$} \\
\hline 1 & 10 & 3 & 17 & 3.5 & 1.7 & 8 & 1 & 0.1312 & 0.1696 & 0.5569 & 0.0124 & 0.8283 & 0.5886 \\
2 & 0.05 & 10 & 17 & 0.1 & 8 & 14 & 1.2 & 0.2329 & 0.4135 & 0.8307 & 0.3736 & 0.1004 & 0.9991 \\
3 & 3 & 3.5 & 1.7 & 10 & 17 & 8 & 3 & 0.2348 & 0.1415 & 0.3522 & 0.2883 & 0.3047 & 0.6650 \\
4 & 17 & 8 & 0.05 & 10 & 0.1 & 14 & 3.2 & 0.4047 & 0.8828 & 0.8732 & 0.5743 & 0.1091 & 0.0381 \\
\hline
\end{tabular}

\section{Shekel's Foxholes Function}

$$
\begin{gathered}
f_{14}(x)=\left[\frac{1}{500}+\sum_{j=1}^{25} \frac{1}{j+\sum_{i=1}^{2}\left(x_{i}-a_{i j}\right)^{6}}\right]^{-1} \\
-65.536 \leq x_{i} \leq 65.536, \quad \min \left(f_{14}\right)=f_{14}(-32,-32) \approx 1
\end{gathered}
$$

where

$\left(a_{i j}\right)=$

$\left(\begin{array}{cccccccccc}-32 & -16 & 0 & 16 & 32 & -32 & \cdots & 0 & 16 & 32 \\ -32 & -32 & -32 & -32 & -32 & -16 & \cdots & 32 & 32 & 32\end{array}\right)$.

\section{N. Kowalik's Function}

$$
\begin{aligned}
f_{15}(x) & =\sum_{i=1}^{11}\left[a_{i}-\frac{x_{1}\left(b_{i}^{2}+b_{i} x_{2}\right)}{b_{i}^{2}+b_{i} x_{3}+x_{4}}\right]^{2} \quad-5 \leq x_{i} \leq 5, \\
\min \left(f_{15}\right) & \approx f_{15}(0.1928,0.1908,0.1231,0.1358) \\
& \approx 0.0003075
\end{aligned}
$$

\section{O. Six-Hump Camel-Back Function}

$$
\begin{aligned}
f_{16}= & 4 x_{1}^{2}-2.1 x_{1}^{4}+\frac{1}{3} x_{1}^{6}+x_{1} x_{2}-4 x_{2}^{2}+4 x_{2}^{4}, \\
& -5 \leq x_{i} \leq 5 \\
x_{\min }= & (0.08983,-0.7126),(-0.08983,0.7126) \\
& \min \left(f_{16}\right)=-1.0316285 .
\end{aligned}
$$

\section{P. Branin Function}

$$
\begin{aligned}
f_{17}(x)= & \left(x_{2}-\frac{5.1}{4 \pi^{2}} x_{1}^{2}+\frac{5}{\pi} x_{1}-6\right)^{2} \\
& +10\left(1-\frac{1}{8 \pi}\right) \cos x_{1}+10 \\
& -5 \leq x_{1} \leq 10, \quad 0 \leq x_{2} \leq 15 \\
x_{\min }= & (-3.14212 .275),(3.142,2.275),(9.425,2.425) \\
& \min \left(f_{17}\right)=0.398
\end{aligned}
$$

\section{Q. Goldstein-Price Function}

$$
\begin{aligned}
f_{18}(x)= & {\left[1+\left(x_{1}+x_{2}+1\right)^{2}\left(19-14 x_{1}+3 x_{1}^{2}-14 x_{2}\right.\right.} \\
& \left.\left.+6 x_{1} x_{2}+3 x_{2}^{2}\right)\right] \times\left[30+\left(2 x_{1}-3 x_{2}\right)^{2}\right. \\
& \left.\times\left(18-32 x_{1}+12 x_{1}^{2}+48 x_{2}-36 x_{1} x_{2}+27 x_{2}^{2}\right)\right] \\
& -2 \leq x_{i} \leq 2, \quad \min \left(f_{18}\right)=f_{18}(0,-1)=3 .
\end{aligned}
$$

TABLE XIV

SHEKEL FunCtIONS $f_{21}, f_{22}, f_{23}$

\begin{tabular}{c|cccc|c}
\hline $\mathrm{i}$ & \multicolumn{4}{|c}{$a_{i j}, j=1, \cdots, 4$} & $c_{i}$ \\
\hline 1 & 4 & 4 & 4 & 4 & 0.1 \\
2 & 1 & 1 & 1 & 1 & 0.2 \\
3 & 8 & 8 & 8 & 8 & 0.2 \\
4 & 6 & 6 & 6 & 6 & 0.4 \\
5 & 3 & 7 & 3 & 7 & 0.4 \\
\hline 6 & 2 & 9 & 2 & 9 & 0.6 \\
7 & 5 & 5 & 3 & 3 & 0.3 \\
\hline 8 & 8 & 1 & 8 & 1 & 0.7 \\
9 & 6 & 2 & 6 & 2 & 0.5 \\
10 & 7 & 3.6 & 7 & 3.6 & 0.5 \\
\hline
\end{tabular}

\section{R. Hartman's Family}

$$
f(x)=-\sum_{i=1}^{4} c_{i} \exp \left[-\sum_{j=1}^{n} a_{i j}\left(x_{j}-p_{i j}\right)^{2}\right]
$$

with $n=3,6$ for $f_{19}(x)$ and $f_{20}(x)$, respectively, $0 \leq$ $x_{j} \leq 1$. The coefficients are defined by Tables XII and XIII, respectively.

For $f_{19}(x)$ the global minimum is equal to -3.86 and it is reached at the point $(0.114,0.556,0.852)$. For $f_{20}(x)$ the global minimum is -3.32 at the point $(0.201,0.150,0.477$, $0.275,0.311,0.657)$.

\section{S. Shekel's Family}

$$
f(x)=-\sum_{i=1}^{m}\left[\left(x-a_{i}\right)\left(x-a_{i}\right)^{T}+c_{i}\right]^{-1}
$$

with $m=5,7$ and 10 for $f_{21}(x), f_{22}(x)$ and $f_{23}(x)$, respectively, $0 \leq x_{j} \leq 10$.

These functions have five, seven, and ten local minima for $f_{21}(x), f_{22}(x)$, and $f_{23}(x)$, respectively. $x_{\text {local_opt }} \approx a_{i}$, $f\left(x_{\text {local_opt }}\right) \approx 1 / c_{i}$ for $1 \leq i \leq m$. The coefficients are defined by Table XIV.

\section{ACKNOWLEDGMENT}

The authors are grateful to anonymous referees and D. B. Fogel for their constructive comments and criticism of earlier versions of this paper, and P. Angeline and T. Bäck for their insightful comments on self-adaptation in evolutionary algorithms. 


\section{REFERENCES}

[1] L. J. Fogel, A. J. Owens, and M. J. Walsh, Artificial Intelligence Through Simulated Evolution. New York: Wiley, 1966.

[2] D. B. Fogel, System Identification Through Simulated Evolution: A Machine Learning Approach to Modeling. Needham Heights, MA: Ginn, 1991.

[3] _ "Evolving artificial intelligence," Ph.D. dissertation, Univ. of California, San Diego, CA, 1992.

[4] _ _ "Applying evolutionary programming to selected traveling salesman problems," Cybern. Syst., vol. 24, pp. 27-36, 1993.

[5] X. Yao, "An overview of evolutionary computation," Chinese J. Adv. Software Res., vol. 3, no. 1, pp. 12-29, 1996.

[6] D. B. Fogel, "An introduction to simulated evolutionary optimization," IEEE Trans. Neural Networks, vol. 5, pp. 3-14, Jan. 1994.

[7] T. Bäck and H.-P. Schwefel, "An overview of evolutionary algorithms for parameter optimization," Evol. Comput., vol. 1, no. 1, pp. 1-23, 1993.

[8] D. B. Fogel, Evolutionary Computation: Toward a New Philosophy of Machine Intelligence. Piscataway, NJ: IEEE Press, 1995.

[9] D. K. Gehlhaar and D. B. Fogel, "Tuning evolutionary programming for conformationally flexible molecular docking," in Evolutionary Programming V: Proc. of the Fifth Annual Conference on Evolutionary Programming, L. J. Fogel, P. J. Angeline, and T. Bäck, Eds. Cambridge, MA: MIT Press, 1996, pp. 419-429.

[10] W. Feller, An Introduction to Probability Theory and Its Applications, vol. 2, 2nd ed. New York: Wiley, 1971.

[11] X. Yao and Y. Liu, "Fast evolutionary programming," in Evolutionary Programming V: Proc. Fifth Annual Conference on Evolutionary Programming, L. J. Fogel, P. J. Angeline, and T. Bäck, Eds. Cambridge, MA: MIT Press, 1996, pp. 451-460.

[12] A. Törn and A. Žilinskas, Global Optimization (Lecture Notes in Computer Science, vol. 350). Berlin, Germany: Springer-Verlag, 1989.

[13] H.-P. Schwefel, Evolution and Optimum Seeking. New York: Wiley, 1995.

[14] D. H. Wolpert and W. G. Macready, "No free lunch theorems for search," Santa Fe Institute, Santa Fe, NM, Tech. Rep. SFI-TR-95-02010,July 1995.

[15] "No free lunch theorems for optimization," IEEE Trans. Evol. Comput., vol. 1, pp. 67-82, Apr. 1997.

[16] L. Devroye, Non-Uniform Random Variate Generation. New York: Springer-Verlag, 1986

[17] R. A. Hunt, Calculus with Analytic Geometry. New York: Harper \& Row, 1986.

[18] X. Yao, "Introduction," Informatica, vol. 18, pp. 375-376, 1994.

[19] K. Chellapilla, "Combining mutation operators in evolutionary programming," IEEE Trans. Evol. Comput., vol. 2, pp. 91-96, Sept. 1998

[20] J. Born, "An evolution strategy with adaptation of the step sizes by a variance function," in Parallel Problem Solving from Nature (PPSN) IV (Lecture Notes in Computer Science, vol. 1141), H.-M. Voigt, W. Ebeling, I. Rechenberg, and H.-P. Schwefel Eds. Berlin, Germany: Springer-Verlag, 1996, pp. 388-397.

[21] C. Kappler, "Are evolutionary algorithms improved by large mutations?," in Parallel Problem Solving from Nature (PPSN) IV (Lecture Notes in Computer Science, vol. 1141), H.-M. Voigt, W. Ebeling, I. Rechenberg, and H.-P. Schwefel Eds. Berlin, Germany: SpringerVerlag, 1996, pp. 346-355.

[22] X. Yao and Y. Liu, "Fast evolution strategies," Contr. Cybern., vol. 26, no. 3, pp. 467-496, 1997.

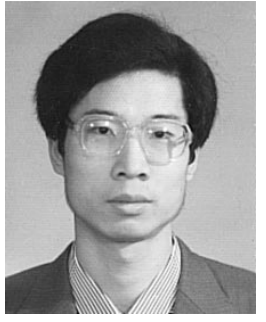

Xin Yao (M'91-SM'96) received the B.Sc. degree from the University of Science and Technology of China (USTC) in 1982, the M.Sc. degree from the North China Institute of Computing Technologies (NCI) in 1985, and the Ph.D. degree from USTC in 1990.

$\mathrm{He}$ is currently a Professor in the School of Computer Science, University of Birmingham, U.K. $\mathrm{He}$ was an Associate Professor in the School of Computer Science, University College, the University of New South Wales, Australian Defence Force Academy (ADFA). He held post-doctoral fellowships in the Australian National University (ANU) and the Commonwealth Scientific and Industrial Research Organization (CSIRO) before joining ADFA in 1992.

Dr. Yao was the Program Committee Cochair for IEEE ICEC'97 and CEC'99 and Co-Vice-Chair for IEEE ICEC'98 in Anchorage. He was also the Program Committee Cochair for SEAL'96, SEAL'98 and ICCIMA'99. He is an Associate Editor of IEEE Transactions on Evolutionary CoMputation and Knowledge and Information Systems: An International Journal (Springer), a member of the editorial board of Journal of Cognitive Systems Research (Elsevier), a member of the IEEE NNC Technical Committee on Evolutionary Computation, and the second vice-president of the Evolutionary Programming Society.

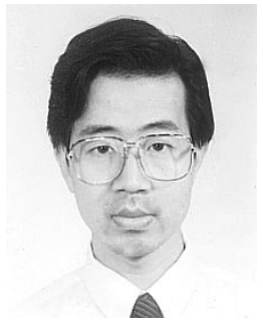

Yong Liu (S'97) received the B.Sc. degree from Wuhan University in 1988 and the M.Sc. degree from Huazhong University of Science and Technology in 1991.

From 1994 to 1996, he was a Lecturer at Wuhan University. Between the end of 1994 to 1995 , he was a Visiting Fellow in the School of Computer Science, University College, the University of New South Wales, Australian Defence Force Academy. $\mathrm{He}$ is currently a Ph.D. candidate in the same school. He has published a number of papers in international journals and conferences and is the first author of the book Genetic Algorithms (Beijing: Science Press, 1995). His research interests include neural networks, evolutionary algorithms, parallel computing, and optimization.

Mr. Liu received the Third Place Award in the 1997 IEEE Region 10 Postgraduate Student Paper Competition.

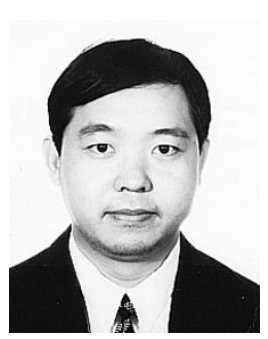

Guangming Lin received the B.Sc. degree in 1985 and the M.Sc. degree in 1988 from Wuhan University, China. He is currently pursuing the $\mathrm{Ph} . \mathrm{D}$. degree in the School of Computer Science, University College, the University of New South Wales, Australian Defence Force Academy, Canberra, Australia.

$\mathrm{He}$ is currently a Lecturer in the Department of Soft Science at Shenzhen University, China. In 1994, he was a Visiting Fellow in the Computer Sciences Laboratory at the Australian National University. He has published a number of papers in the fields of evolutionary computation and parallel computing. His research interests include evolutionary algorithms, parallel computing and optimization. 